\title{
Remarks on Boson Commutation Rules
}

\author{
C. ITZYKSON \\ Service de Physique Théorique \\ Centre d'Etudes Nucléaires de Saclay \\ BP No $2-91$ Gif-sur-Yvette
}

Received July 15, 1966

\begin{abstract}
A group theoretical derivation is given of Bargmann's representation of the boson commutation rules in an Hilbert space of analytic functions. Several interesting problems arise in the study of the global representation of the canonical group $S_{p}(2 n, R)$. As a by-product we recover Laguerre-polynomials as spherical functions on the nilpotent Weyl group.
\end{abstract}

\section{Introduction}

Several years ago, V. Bargmann [1] has described a representation of the creation and annihilation operators for bosons in a Hilbert space of analytic functions. We want to show some interesting connections between this construction and the theory of group representations. This appears when one attempts to find a representation of the basic commutation rules of quantum mechanics $[p, q]=-i$ using the device of WeYL. Namely, one introduces a nilpotent group the Lie algebra of which is closely related to the preceding commutation relations. It will be shown that this leads quite naturally to the Bargmann space of entire functions. One may then ask for the global representation of the canonical group in this space. Again this was done by Baramann though not in great detail. We observe that the representation splits into two irreducible parts (a fact well known in the study of the harmonic oscillator) each of which is double valued. This appears to have been noticed only recently [2] and it is amusing to note the analogy with the fermion case. This is somehow unexpected, since in contrast with the orthogonal real groups the symplectic ones have an infinitely sheeted covering.

The study of the canonical transformations is made much easier by a mapping on a second Hilbert space of analytic functions in a unit disk. It allows to compare the double valued representation to the known onevalued ones of $S L(2, R)[3]$.

One can easily extend these considerations to $N$ degrees of freedom. The analog of the unit disk is found in this case to be the set of $N$ by $N$ complex symmetric matrices $S$ such that the hermitian matrix $I-\bar{S} S$ is positive definite. We shall denote the set of these matrices by $\mathscr{S}_{N}$. 
We shall need repeatedly the following integral [1]

$$
\int_{R^{n}} \exp \{-T(x)+2 b \cdot x\} d^{n} x=\pi^{n / 2}(\operatorname{det} T)^{-1 / 2} \exp \left\{T^{-1}(b)\right\},
$$

where the notations are as follows: $T(x)$ is a quadratic form, $T^{-1}$ its inverse; the integral converges absolutely if and only if the real part of $T$ is positive definite. The determination of $(\operatorname{det} T)^{-1 / 2}$ is obtained by the analytic continuation of $(\operatorname{det} T(\alpha))^{-1 / 2}$ with $T(\alpha)=T^{\prime}+i \alpha T^{\prime \prime}$, ( $T^{\prime}$ and $T^{\prime \prime}$ real and imaginary parts of $T$ ) from a positive value of $(\operatorname{det} T(0))^{-1 / 2}$ and $0 \leqq \alpha \leqq 1$.

Many thanks are due to Dr. R. SToRA for stimulating discussions on the subject. He has pointed out that in VILENKrN's book "Special functions and theory of group representations" Moscow (1965) a similar connection between the nilpotent Weyl group and Laguerre polynomials is to be found as in section II. Also a recent preprint by R. F. STREATER "The representations of the oscillator group" (Imperial College preprint, March 1966) has some relation with the present work.

\section{Representations of a nilpotent group}

Consider the set of 3 by 3 triangular matrices

$$
h(\alpha, \beta, \gamma)=\left(\begin{array}{lll}
1 & 0 & 0 \\
\alpha & 1 & 0 \\
\beta & \gamma & 1
\end{array}\right)
$$

with $\alpha, \beta, \gamma$ complex numbers. Clearly these matrices form a Lie group. The multiplication law reads:

$$
h(\alpha, \beta, \gamma) h\left(\alpha^{\prime}, \beta^{\prime}, \gamma^{\prime}\right)=h\left(\alpha+\alpha^{\prime}, \beta+\beta^{\prime}+\gamma \alpha^{\prime}, \gamma+\gamma^{\prime}\right) .
$$

The Lie algebra in terms of 3 by 3 matrices is spanned by :

Indeed

$$
\begin{gathered}
a_{1}=\left(\begin{array}{lll}
0 & 0 & 0 \\
1 & 0 & 0 \\
0 & 0 & 0
\end{array}\right) a_{2}=\left(\begin{array}{lll}
0 & 0 & 0 \\
0 & 0 & 0 \\
0 & 1 & 0
\end{array}\right) a_{3}=\left(\begin{array}{lll}
0 & 0 & 0 \\
0 & 0 & 0 \\
1 & 0 & 0
\end{array}\right) \\
{\left[a_{1}, a_{2}\right]=-a_{3} \quad\left[a_{1}, a_{3}\right]=0 \quad\left[a_{2}, a_{3}\right]=0 .}
\end{gathered}
$$

$$
\exp \left\{\varepsilon_{1} a_{1}+\varepsilon_{2} a_{2}+\varepsilon_{3} a_{3}\right\}=h\left(\varepsilon_{1}, \frac{\varepsilon_{1} \varepsilon_{2}}{2}+\varepsilon_{3}, \varepsilon_{2}\right)
$$

with $\varepsilon_{i}$ three complex numbers. The Lie algebra (3) is nilpotent since in the adjoint representation

$$
\left[\operatorname{adj}\left(\varepsilon_{1} a_{1}+\varepsilon_{2} a_{2}+\varepsilon_{3} a_{3}\right)\right]^{2}=0 .
$$

We are interested in a real form of the group considered above, namely the case $\gamma=-\bar{\alpha}$ and $\beta=-\frac{\alpha \bar{\alpha}}{2}+i \varrho$, i.e.

$$
g(\alpha, \varrho)=h\left(\alpha,-\frac{\alpha \bar{\alpha}}{2}+i \varrho,-\bar{\alpha}\right)=\left(\begin{array}{ccc}
1 & 0 & 0 \\
\alpha & 1 & 0 \\
-\frac{\alpha \bar{\alpha}}{2}+i \varrho & -\bar{\alpha} & 1
\end{array}\right)
$$

7 Commun. math. Phys., Vol. 4 
where we use $\bar{\alpha}$ to mean the conjugate of the complex number $\alpha$. That our restrictions are compatible with the group law (2) is readily verified since

$$
\begin{gathered}
\operatorname{Re}\left[\left(-\frac{\alpha \bar{\alpha}}{2}+i \varrho\right)+\left(-\frac{\alpha^{\prime} \bar{\alpha}^{\prime}}{2}+i \varrho^{\prime}\right)-\bar{\alpha} \alpha^{\prime}\right]=-\frac{1}{2}\left(\alpha \bar{\alpha}+\alpha^{\prime} \bar{\alpha}^{\prime}+\bar{\alpha} \alpha^{\prime}+\bar{\alpha}^{\prime} \alpha\right) \\
=-\frac{1}{2}\left(\alpha+\alpha^{\prime}\right)\left(\bar{\alpha}+\bar{\alpha}^{\prime}\right) .
\end{gathered}
$$

From (2) we deduce:

$$
\left\{\begin{array}{l}
g(\alpha, \varrho) g\left(\alpha^{\prime}, \varrho^{\prime}\right)=g\left(\alpha+\alpha^{\prime}, \varrho+\varrho^{\prime}+\frac{\alpha \bar{\alpha}^{\prime}-\bar{\alpha} \alpha^{\prime}}{2 i}\right) \\
\alpha \text { complex, }{ }^{\prime} \text { real } .
\end{array}\right.
$$

This group we shall call the Weyl group $W$. It is a 3 real parameter Lie group. As a manifold, it is isomorphic with a 3 -dimensional real euclidian space with an invariant measure proportional to $d^{2} \alpha d \varrho$, where $d^{2} \alpha$ stands for $d \operatorname{Re} \alpha d \operatorname{Im} \alpha$. In the 3 by 3 representation given by (4) the Lie algebra is again given by the matrices $a_{1}, a_{2}, a_{3}$ but the restriction on the coefficients yields $\varepsilon_{2}=-\bar{\varepsilon}_{1} \varepsilon_{3}=-\bar{\varepsilon}_{3}$. It is then recognized that the nilpotent Lie algebra of $W$ is isomorphic with that provided by the usual commutation rules $[p, q]=\frac{h I}{i},[p, I]=[q, I]=0$.

We then proceed to build a series of unitary representations of $W$ using the method of induced representations. For that purpose, we select in $W$ a subgroup $W_{\alpha_{0}} \subset W$ of those $g$ that can be written:

$W_{\alpha_{0}}:\left\{g\left(\alpha_{0} u, v\right) u\right.$ and $v$ real, $\alpha_{0} \neq 0$ fixed complex number $\}$.

Clearly $W_{\alpha_{0}}$ is a two parameter abelian group since:

$$
g\left(\alpha_{0} u, v\right) g\left(\alpha_{0} u^{\prime}, v^{\prime}\right)=g\left(\alpha_{0}\left(u+u^{\prime}\right), v+v^{\prime}\right) .
$$

The cosets $g W_{\alpha_{0}}$ can be parametrized in terms of one real parameter $w$ in such a way that in each coset there is one and only one element of the form $g\left(i \alpha_{0} w, 0\right)$. Indeed, for $\alpha$ and $\varrho$ arbitrary, the decomposition reads:

$$
g(\alpha, \varrho)=g\left(i \alpha_{0} \operatorname{Im} \frac{\alpha}{\alpha_{0}}, 0\right) g\left(\alpha_{0} \operatorname{Re} \frac{\alpha}{\alpha_{0}}, \varrho-\frac{\alpha_{0} \bar{\alpha}_{0}}{2} \operatorname{Im}\left(\frac{\alpha}{\alpha_{0}}\right)^{2}\right) .
$$

The abelian group $W_{\alpha_{0}}$ has one dimensional unitary representations

$$
g\left(\alpha_{0} u, v\right) \rightarrow e^{i(\mu u+v v)}
$$

where $\mu$ and $v$ real label the representation. We then introduce the set of functions $\{F\}$ defined on $W$ with the property that:

$$
F\left(g g\left(\alpha_{0} u, v\right)\right)=e^{-i(\mu u+v v)} F(g) .
$$

In view of (7) and (8) $F$ is entirely known once its value is given for the elements $g\left(i \alpha_{0} w, 0\right)$.

To each $F$ we then associate a function of one variable

$$
f(w)=F\left(g\left(i \alpha_{0} w, 0\right)\right) .
$$


The group $W$ acts on the functions $F$ through

$$
F \rightarrow{ }^{g_{1}} F \quad g_{1} F(g)=F\left(g_{1}^{-1} g\right) ;
$$

this can be carried over to $f$ with the help of (9). All we need is to find the function ${ }^{g_{1}} f$ associated to ${ }^{g_{1}} F$. We proceed as follows. Let $g_{1}=(\alpha, \varrho)$, then $g_{1}^{-1}=(-\alpha,-\varrho)$ and from (5) and (7)

$g_{1}^{-1} \cdot g\left(i \alpha_{0} w, 0\right)=g\left(i \alpha_{0} w-\alpha,-\varrho+w \operatorname{Re} \alpha \bar{\alpha}_{0}\right)$

$$
\begin{aligned}
= & g\left(i \alpha_{0}\left(w-\operatorname{Im} \frac{\alpha}{\alpha_{0}}\right), 0\right) \times \\
& \times g\left(-\alpha_{0} \operatorname{Re} \frac{\alpha}{\alpha_{0}},-\varrho+2 w \operatorname{Re} \alpha \bar{\alpha}_{0}-\frac{\alpha_{0} \bar{\alpha}_{0}}{2} \operatorname{Im}\left(\frac{\alpha}{\alpha_{0}}\right)^{2}\right) .
\end{aligned}
$$

Using this result:

$$
\begin{gathered}
g_{1} f(w)={ }^{g_{1}} F\left(g\left(i \alpha_{0} w, 0\right)\right)=F\left(g_{1}^{-1} \cdot g\left(i \alpha_{0} w, 0\right)\right) \\
\left\{\begin{array}{l}
g_{1} f(w)=e^{i\left[\mu \operatorname{Re} \frac{\alpha}{\alpha_{0}}+v\left(\varrho-2 w \operatorname{Re} \alpha \bar{\alpha}_{0}+\frac{1}{2} \alpha_{0} \bar{\alpha}_{0} \operatorname{Im}\left(\frac{\alpha}{\alpha_{0}}\right)^{2}\right)\right]} f\left(w-\operatorname{Im} \frac{\alpha}{\alpha_{0}}\right) \\
g_{1} \equiv g_{1}(\alpha, \varrho) .
\end{array}\right.
\end{gathered}
$$

The group law is easily verified.

Equation (10) gives us a set of representations of the group $W$ on functions of one variable. Clearly if

$$
\|f\|^{2}=\int_{-\infty}^{+\infty} d w|f(w)|^{2}<\infty, \text { then }\left\|{ }^{g_{1}} f\right\|=\|f\|,
$$

i.e. these representations are unitary. The case $v=0$ is somewhat singular. The representation is not faithfull. It reduces to a direct integral. Except in that case the representation is irreducible. If $f$ is differentiable and such that its derivative is square integrable, we can obtain the infinitesimal form of (10). Assuming $\alpha$ and $\varrho$ very small

$$
\begin{aligned}
g_{1} f(w) \cong & {\left[1+i \mu \operatorname{Re} \frac{\alpha}{\alpha_{0}}+i \nu \varrho-\operatorname{Im} \frac{\alpha}{\alpha_{0}} \frac{\partial}{\partial w}-2 i \nu w \operatorname{Re} \alpha \bar{\alpha}_{0}\right] f(w) \cong } \\
\cong & {\left[1+i \nu \varrho+\alpha \frac{i}{2 \alpha_{0}}\left(\mu+\frac{\partial}{\partial w}-2 \nu w \alpha_{0} \bar{\alpha}_{0}\right)-\right.} \\
& \left.-\bar{\alpha} \frac{i}{2 \bar{\alpha}_{0}}\left(-\mu+\frac{\partial}{\partial w}+2 \nu w \alpha_{0} \bar{\alpha}_{0}\right)\right] f(w) .
\end{aligned}
$$

It will be convenient to identify this with infinitesimal generators defined through

Hence, we get:

$$
{ }^{g_{1}} f(w) \cong\left(1+i \varrho B+\alpha A^{+}-\bar{\alpha} A\right) f(w) .
$$

$$
\left\{\begin{array}{l}
A^{+}=\frac{i}{2 \alpha_{0}}\left[\mu+\frac{\partial}{\partial w}-2 v w \alpha_{0} \bar{\alpha}_{0}\right] \\
A=\frac{i}{2 \bar{\alpha}_{0}}\left[-\mu+\frac{\partial}{\partial w}+2 \nu w \alpha_{0} \bar{\alpha}_{0}\right] \\
B=\nu I
\end{array}\right.
$$


with

$$
\left[A, A^{+}\right]=B,[A, B]=\left[A^{+}, B\right]=0 .
$$

In particular, if we set $\alpha_{0}=-\frac{i}{\sqrt{2}}, \mu=0, \nu=1$, we get the usual representation of quantum mechanics on the space $\mathscr{L}^{2}(\mathbb{R})$ of square integrable functions of one variable. In the following, we stick to these particular values:

$$
\alpha_{0}=\frac{-i}{\sqrt{2}}, \mu=0, v=1
$$

Then the group acts according to

$$
\left\{\begin{array}{l}
g=g(\alpha, \varrho) \\
g f(w)=e^{i\left(\varrho+\sqrt{2} w \operatorname{Im} \alpha-\frac{1}{2} \operatorname{Im} \alpha^{2}\right)} f(w-\sqrt{2} \operatorname{Re} \alpha) .
\end{array}\right.
$$

We can regard equation $\left(10^{\prime}\right)$ for fixed $w$ as defining a certain function on the group manifold. To be more precise, we restrict our attention to those $f \in \mathscr{L}^{2}(\mathbb{R})$ which are infinitely differentiable, then for each $w \in \mathbb{R}$ equation $\left(10^{\prime}\right)$ gives a mapping $f \stackrel{w}{\longrightarrow} f(g)={ }^{g} f(w)$ leading to an infinitely differentiable function on $W$. $W$ itself can be considered as product of a real line $(\varrho)$ times a complex plane $(\alpha)$. It is tempting to try to see whether there exist functions $f$ such that $f$ be analytic in $\alpha$ for fixed $\varrho$. Actually this does not work but instead one has the following result: there exists up to a factor one and only one infinitely differentiable function $f(w)$ such that $f(g)$ given by $\left(10^{\prime}\right)$ is equal to $e^{\frac{-\alpha \bar{\alpha}}{2}}$ times an analytic function of $\alpha$. To see this we solve

This reads:

$$
\frac{\partial}{\partial \bar{\alpha}} e^{\frac{\alpha \bar{\alpha}}{2}+i\left(\sqrt{2} w \operatorname{Im} \alpha-\frac{1}{2} \operatorname{Im} \alpha^{2}\right)} f(w-\sqrt{2} \operatorname{Re} \alpha)=0 .
$$

$$
\left(\operatorname{Re} \alpha-\frac{w}{\sqrt{2}}\right) f(w-\sqrt{2} \operatorname{Re} \alpha)-\frac{1}{\sqrt{2}} f^{\prime}(w-\sqrt{2} \operatorname{Re} \alpha)=0
$$

or

$$
w f(w)+f^{\prime}(w)=0
$$

hence

$$
f(w)=\operatorname{cte} e^{-\frac{w^{2}}{2}}
$$

With the proper normalization we call this function:

$$
f_{0}(w)=\pi^{-\frac{1}{4}} e^{-\frac{w^{2}}{2}} .
$$

For $g$ of the form $g(\alpha, 0)$ we get from $\left(10^{\prime}\right)$ :

$$
\left\{\begin{aligned}
{ }^{g} f_{0}(w) & =\pi^{-\frac{1}{4}} \exp \left\{-\frac{w^{2}}{2}-\frac{1}{2} \alpha \bar{\alpha}+\sqrt{2} w \alpha-\frac{1}{2} \alpha^{2}\right\} \\
& =\pi^{-\frac{1}{4}} \exp \left\{-\frac{w^{2}}{2}-\frac{1}{2} \alpha \bar{\alpha}\right\} \sum_{0}^{\infty} \frac{\alpha^{n}}{\sqrt{n !}} \frac{1}{\sqrt{2^{n} n !}} H_{n}(w),
\end{aligned}\right.
$$


where we have recognized in (15) the generating function for Hermite polynomials. We are now in position to introduce the Bargmann space. For two square integrable functions $f_{1}$ and $f_{2}$ let us denote by $\left(f_{1}, f_{2}\right)$ their scalar product

$$
\left(f_{1}, f_{2}\right)=\int_{-\infty}^{+\infty} \overline{f_{1}}(w) f_{2}(w) d w
$$

Then to each square integrable $f$ we associate the analytic function $H f$ :

$$
\left\{\begin{array}{l}
\left.H f(\alpha)=e^{\frac{\alpha \bar{\alpha}}{2}(g(\bar{\alpha}, 0)} f_{0}, f\right) \\
=\pi^{-\frac{1}{4}} \int_{-\infty}^{+\infty} d w e^{-\frac{1}{2} \alpha^{2}-\frac{w^{2}}{2}+\sqrt{2} w \alpha} f(w), \quad f \in \mathscr{L}^{2}(R) .
\end{array}\right.
$$

We shall see below that equation (16) has a second interesting interpretation. This functional space has been studied in detail in reference [1] and we shall sketch very briefly the results we need. Let $f(w)$ be expanded in terms of a complete set of orthonormalized Hermite functions

$$
f(w)=\sum_{0}^{\infty} c_{n}\left(e^{-\frac{w_{2}}{2}} \frac{\pi^{-\frac{1}{4}}}{\sqrt{2^{n} n !}} H_{n}(w)\right) \quad\|f\|^{2}=\sum_{0}^{\infty}\left|c_{n}\right|^{2},
$$

the convergence being understood in norm; then one deduces from (15) and (16) that:

$$
H f(\alpha)=\sum_{0}^{\infty} c_{n} \frac{\alpha^{n}}{\sqrt{n !}} \text { is an entire analytic function. }
$$

It is then natural to define a scalar product by

$$
\left(H f_{1}, H f_{2}\right)_{\mathscr{B}}=\left(f_{1}, f_{2}\right)
$$

where the subscript $\mathscr{B}$ indicates that we evaluate a scalar product in a new space. The operator $H$ is isometric. One shows that $\left(H f_{1}, H f_{2}\right)_{\mathscr{B}}$ can be written as an integral

$$
\begin{gathered}
\left(H f_{1}, H f_{2}\right)_{\mathscr{B}}=\int d \mu(\alpha) \overline{H f_{1}(\alpha)} \cdot H f_{2}(\alpha)=\left(f_{1}, f_{2}\right), \\
d \mu(\alpha)=\frac{d \operatorname{Re} \alpha d \operatorname{Im} \alpha e^{-\alpha \bar{\alpha}}}{\pi} .
\end{gathered}
$$

The analytic functions with finite norm (defined through (17)) build a Hilbert space and $H$ is then unitary. Let us call this Hilbert space $\mathscr{B}$.

The group $W$ acts on $\mathscr{B}$ by requiring that

This gives with $g \equiv g(\alpha, \varrho)$

$$
{ }^{g} H f=H^{g} f \text {. }
$$

$$
\begin{gathered}
{ }^{g} H f(z)=e^{\frac{z \bar{z}}{2}}\left({ }^{g(\bar{z}, 0)} f_{0},{ }^{g} f\right)=e^{\frac{z \bar{z}}{2}}\left(g^{g-1 \cdot g(\bar{z}, 0)} f_{0}, f\right) \\
g(\alpha, e) H f(z)=e^{i \varrho+\alpha z-\frac{\alpha \bar{\alpha}}{2}} H f(z-\bar{\alpha}) .
\end{gathered}
$$


In deriving (19) we have used the fact that $g$ acts unitarily on $\mathscr{L}^{2}(\mathbb{R})$. From the infinitesimal form of (19) we get the representation in $\mathscr{B}$ of the Lie algebra of $W$ (compare with (11)):

hence

$$
g(\alpha, \varrho) H f(z) \cong\left(1+i \varrho+\alpha z-\bar{\alpha} \frac{\partial}{\partial z}\right) H f(z),
$$

$$
A=\frac{\partial}{\partial z} \quad A^{+}=z \quad B=I
$$

We can also deduce the image in $\mathscr{B}$ of $f_{0}$. From (15) and (16):

$$
H f_{0}(z)=F_{0}(z)=e^{\frac{\alpha \bar{\alpha}}{2}}\left({ }^{g(\bar{\alpha}, 0)} f_{0}, f_{0}\right)=1
$$

and from (19)

$$
{ }^{g(\bar{\alpha}, 0)} F_{0}(z)=F_{\alpha}(z)=e^{\bar{\alpha} z-\frac{\alpha \bar{\alpha}}{2}} .
$$

We can now give a second interpretation of (16) as follows. Let $F \in \mathscr{B}$, then it is the image under $H$ of some $f \in \mathscr{L}^{2}(\mathbb{R})$; according to (17) and (18)

$$
\left.{ }^{(g(\bar{\alpha}, 0)} f_{n}, f\right)=\left(H^{g(\bar{\alpha}, 0)} f_{0}, H f\right)_{\mathscr{B}}=\left({ }^{g(\bar{\alpha}, 0)} H f_{0}, H f\right)_{\mathscr{B}} .
$$

Now ${ }^{g(\bar{\alpha}, 0)} H f_{0}=F_{\alpha}(z)$ as expressed by $(21), H f=F$ and (16) also reads:

$$
F(\alpha)=e^{\frac{\alpha \bar{\alpha}}{2}}\left(F_{\alpha}, F\right)_{\mathscr{B}}=\int d \mu(z) e^{\alpha \bar{z}} F(z),
$$

so that $e^{\frac{\alpha \alpha}{2}} F_{\alpha}(z)=e^{\bar{\alpha} z}$ plays the role of a "delta function" in the Hilbert space $\mathscr{B}$. Moreover $f_{0}$ was of unit norm and $g$ acts unitarily, hence $\left\|F_{\alpha}\right\|=1$, and Schwarz inequality applied to (22) leads to

$$
|F(\alpha)| \leqq\|F\| e^{\frac{\alpha \bar{\alpha}}{2}} .
$$

The detailed mathematical analysis of the space $\mathscr{B}$ and of the mapping $H$ are given by BARGMANN in [1]; we have merely pointed out that the whole construction can be given a rather detailed group theoretic interpretation. It is also worth mentioning that one can directly arrive to $\mathscr{B}$ if one induces representations of $W$ by selecting only the one parameter abelian subgroup $W_{0}$ of the elements of the form $g(0, \varrho)$. But one looses this way the connection with the usual representation in terms of square integrable function of one variable.

We complete this section by computing matrix elements of $W$. We have seen previously that the functions

$$
b_{m}(z)=\frac{z^{m}}{\sqrt{m !}} m=0,1, \ldots
$$

make up a complete orthonormal basis in the Hilbert space $\mathscr{B}$. Let us 
compute

$$
\begin{gathered}
U_{n, m}(\alpha, \varrho)=\left(b_{n}, g(\alpha, \varrho) b_{m}\right)_{\mathscr{B}} \\
g(\alpha, \varrho) b_{m}(z)=\frac{1}{\sqrt{m !}} e^{i \varrho+\alpha z-\frac{\alpha \bar{\alpha}}{2}}(z-\bar{\alpha})^{m} \\
=e^{i \varrho-\frac{\alpha \bar{\alpha}}{2}} \sqrt{m !} \sum_{s=0}^{\infty} \sum_{t=0}^{m} z^{s+t} \frac{\alpha^{s}(-\bar{\alpha})^{m-t}}{s ! t !(m-t) !} \\
U_{n, m}(\alpha, \varrho)=e^{i \varrho-\frac{\alpha \bar{\alpha}}{2}} \sqrt{n ! m !} \sum_{t}^{\prime} \frac{\alpha^{n-t}(-\bar{\alpha})^{m-t}}{(n-t) !(m-t) ! t !}
\end{gathered}
$$

where $\Sigma^{\prime}$ means that the sum has to be taken for non-negative arguments of the factorials. Now recall that the associated Laguerre polynomials are defined by

$$
\begin{gathered}
L^{k}{ }_{p}(x)=\sum_{s=0}^{p} \frac{(-x)^{s}}{s !} \frac{\Gamma(p+k+1)}{(p-s) ! \Gamma(k+s+1)} \\
\int_{0}^{\infty} d x e^{-x} x^{k} L_{p}^{k}(x) L_{q}^{k}(x)=\frac{\Gamma(p+k+1)}{p !} \delta_{p, q} \\
k, p=0,1, \ldots
\end{gathered}
$$

it turns out to be useful to define $L_{p}^{k}(x)$ also for negative integer $k$ through:

$$
\frac{1}{m !} L_{m+k}^{-k}(x)=\frac{1}{(m+k) !}(-x)^{k} L_{m}^{k}(x) .
$$

This definition is in agreement with our expression of $L_{p}^{k}(x)$ given above provided one remarks that $\frac{1}{\Gamma(k+s+1)}=0$ for $s \leqq-k-1, s$ integer. This enables one to write

$$
U_{n m}(\alpha, \varrho)=\sqrt{\frac{m !}{n !}} e^{i \varrho-\frac{\bar{\alpha} \alpha}{2}} \alpha^{n-m} L_{m}^{n-m}(\alpha \bar{\alpha}) .
$$

One verifies of course the unitarity condition $U_{n, m}(\alpha, \varrho)=\overline{U_{m, n}(-\alpha,-\varrho)}$ and the orthogonality relations

$$
\int \frac{d^{2} \alpha}{\pi} \overline{U_{n, m}(\alpha, 0)} U_{n^{\prime}, m^{\prime}}(\alpha, 0)=\delta_{n, n^{\prime}} \delta_{m, m^{\prime}} .
$$

From the group law $U(g) U\left(g^{\prime}\right)=U\left(g g^{\prime}\right)$ one also deduces

$$
\begin{aligned}
e^{-\bar{\alpha}_{1} \alpha_{2}}\left(\alpha_{1}+\alpha_{2}\right)^{n-m} L_{m}^{n-m} & {\left[\left(\alpha_{1}+\alpha_{2}\right)\left(\bar{\alpha}_{1}+\bar{\alpha}_{2}\right)\right] } \\
= & \sum_{s=0}^{\infty} \alpha_{1}^{n-s} \alpha_{2}^{s-m} L_{s}^{n-s}\left(\alpha_{1} \bar{\alpha}_{1}\right) L_{m}^{s-m}\left(\alpha_{2} \bar{\alpha}_{2}\right)
\end{aligned}
$$

The character of the representation is given by:

$$
\begin{aligned}
& \chi(\alpha, \varrho)=\sum_{n=0}^{\infty} U_{n, n}(\alpha, \varrho)=e^{i \varrho} \int d \mu(z) e^{+\alpha z-\frac{\alpha \bar{\alpha}}{2}} \sum_{n=0}^{\infty} \frac{(z-\bar{\alpha})^{n} \bar{z}^{n}}{n !} \\
& =e^{i \varrho} \int \frac{d^{2} z}{\pi} e^{-\frac{\alpha \bar{\alpha}}{2}+\alpha z-\bar{\alpha} \bar{z}}=e^{i \varrho} \delta^{(2)}(\alpha),
\end{aligned}
$$


where $\delta^{(2)}(\alpha)$ stands for $\delta(\operatorname{Re} \alpha) \delta(\operatorname{Im} \alpha)$. The preceding formulae can be used to derive numerous properties of Laguerre polynomials. We give a few examples in appendix I.

\section{Canonical transformations}

Our aim in this section is to study certain automorphisms of the group $W$. To be more precise we are interested by the continuous automorphisms of $W$ of the type

$$
g(\alpha, \varrho) \rightarrow g(\hat{\alpha}, \varrho) \equiv \hat{g}
$$

with $\hat{\alpha}$ function of $\alpha$. The conditions to be satisfied by this correspondance to be an automorphism are

(i) $\widehat{\alpha+\beta}=\hat{\alpha}+\hat{\beta}$,

(ii) $\operatorname{Im} \hat{\alpha} \bar{\beta}=\operatorname{Im} \alpha \bar{\beta}$.

The only continuous solutions of (i) are $\hat{\alpha}=\bar{\lambda} \alpha-\mu \bar{\alpha}$, while (ii) imposes $|\lambda|^{2}-|\mu|^{2}=1$ which also insures the mapping to be one to one. The notation is chosen for convenience. These automorphisms are in one to one correspondance with the matrices

$$
k=\left(\begin{array}{cc}
\bar{\lambda} & -\mu \\
-\bar{\mu} & \lambda
\end{array}\right)
$$

and to the product of automorphisms corresponds the product of the matrices. These form a group isomorphic to the special linear real group in two dimensions $S L(2, R)$ which we shall denote in the following by $K$, while ${ }^{k} g$ will stand for the transform under $k \in K$ of an element $g \in W . K$ is the canonical group. Its action on the generators of $W$ is defined through:

That is:

$$
\alpha^{k} A^{+}-\bar{\alpha}^{k} A=(\bar{\lambda} \alpha-\mu \bar{\alpha}) A^{+}-(\lambda \bar{\alpha}-\bar{\mu} \alpha) A .
$$

$$
\begin{aligned}
{ }^{k} A^{+} & =\bar{\lambda} A^{+}+\bar{\mu} A \\
{ }^{k} A & =\lambda A+\mu A^{+} .
\end{aligned}
$$

We now seek in the space $\mathscr{B}$ a set of operators $V(k)$ such that:

$$
U\left({ }^{k} g\right)=V(k) U(g) V^{-1}(k) .
$$

In order to solve (29) we shall follow reference [1]. We remark that in $\mathscr{B}$ we had a set of vectors $F_{t}(z)=e^{z t-\frac{t \bar{t}}{2}}$ which allows one to compute the value of any function $F \in \mathscr{B}$ at the point $t$ through $F(t)=e^{\frac{t \bar{t}}{2}}\left(F_{t}, F^{\prime}\right)$. 
They are defined except for a factor by

$$
(A-\bar{t}) F_{t}=0 \text {. }
$$

Now according to (29) one requires that

$$
\left({ }^{k} A-\bar{t}\right) V(k) F_{t}=0
$$

or

$$
\left(\lambda \frac{\partial}{\partial z}+\mu z-\bar{t}\right) V(k) F_{t}(z)=0,
$$

so that, we can choose:

$$
\left\{\begin{array}{l}
V(k) F_{t}(z)=\frac{1}{\sqrt{\lambda}} \exp \left\{-\frac{t \bar{t}}{2}+\frac{\bar{\mu}}{2 \lambda} \bar{t}^{2}-\frac{\mu}{2 \lambda} z^{2}+\frac{\bar{t}}{\lambda} z\right\} \\
\sqrt{\lambda}=\sqrt{|\lambda|} e^{\frac{1}{2} \operatorname{Arg} \lambda}-\pi \leqq \operatorname{Arg} \lambda<\pi .
\end{array}\right.
$$

The various factors in $(30)$ are such that $\left\|V(k) F_{t}\right\|=\left\|F_{t}\right\|=1$. One can check this with the help of equation (1).

Hence we define $V(k)$ for arbitrary $F \in \mathscr{B}$ through the absolutely convergent integral:

$$
\left\{\begin{array}{l}
V(k) F(t)=\lambda^{-\frac{1}{2}} \int d \mu(z) F(z) \exp \left\{\frac{\bar{\mu}}{2 \lambda} \bar{z}^{2}+\frac{t}{\lambda} \bar{z}-\frac{\mu}{2 \lambda} t^{2}\right\} \\
\sqrt{\lambda}=\sqrt{|\lambda|} e^{\frac{1}{2} \operatorname{Arg} \lambda}-\pi \leqq \operatorname{Arg} \lambda<\pi .
\end{array}\right.
$$

A tedious though straightforward calculation shows that:

(i) $V(k)$ is unitary,

(ii) the condition (29) is indeed satisfied,

(iii) the result for $F_{t}(z)$ is the one given above.

The operators $V(k)$ satisfy obviously the following indentity

$$
\left[V(k) V\left(k^{\prime}\right) V^{-1}\left(k k^{\prime}\right), U(g)\right]=0
$$

whatever $g$. Since the representation $U(g)$ is irreducible, we expect $V(k) V\left(k^{\prime}\right)$ to differ from $V\left(k k^{\prime}\right)$ only perhaps by a phase factor. That is they generate a projective representation of the canonical group $K$. In fact we have choosen the phases in (31) in such a way that the arbitrariness be at most a sign; this is reflected in the $\lambda^{-\frac{1}{2}}$ which appears in front of the integral. We want to make this more explicit. The manifold of the group $K$ is topologically isomorphic to the product of a twodimensional plane and a circle. This is obvious if we choose as parametrization: $\mu$ arbitrary complex number and $\lambda=e^{i \Phi} \sqrt{1+|\mu|^{2}}$. Hence the universal covering is infinitely sheeted. Equation (31) provides us with a representation of a two sheeted covering group of $K$. 
The analysis of the representation of the Lie algebra of $K$ is simple. Let $k$ be close to unity we write:

$$
\left\{\begin{array}{l}
k \cong I-\left(i 2 \delta_{1} M_{0}+\delta_{2} M_{+}-\bar{\delta}_{2} M_{-}\right) \\
\delta_{1} \text { real, } \quad \delta_{2} \text { complex } M_{0}=\frac{1}{2}\left(\begin{array}{ll}
1 & 0 \\
0 & -1
\end{array}\right), \quad M_{+}=\left(\begin{array}{ll}
0 & 1 \\
0 & 0
\end{array}\right), \quad M_{-}=\left(\begin{array}{rr}
0 & 0 \\
-1 & 0
\end{array}\right) \\
\text { with }\left[M_{0}, M_{+}\right]=M_{+} \\
{\left[M_{0}, M_{-}\right]=-M_{-}} \\
{\left[M_{+}, M_{-}\right]=-2 M_{0}}
\end{array}\right.
$$

and $\lambda \cong e^{i \delta_{1}} \mu \cong \delta_{2}$. Using in $\mathscr{B}$ the dense subset of polynomials on which the Lie algebra can apply without restrictions (31) yields

$$
\left\{\begin{array}{l}
V(k) F(z) \cong\left[1-i \delta_{1}\left(\frac{1}{2}+z \frac{\partial}{\partial z}\right)-\delta_{2} \frac{z^{2}}{2}+\bar{\delta}_{2} \frac{1}{2} \frac{\partial^{2}}{\partial z^{2}}\right] F(z) \\
\text { or } \quad V\left(M_{0}\right)=\frac{1}{2}\left(\frac{1}{2}+A^{+} A\right) \quad V\left(M_{+}\right)=\frac{1}{2} A^{+2} \quad V\left(M_{-}\right)=\frac{1}{2} A^{2}
\end{array}\right.
$$

$V\left(M_{0}\right), V\left(M_{+}\right)+V\left(M_{-}\right)$and $\frac{1}{i}\left[V\left(M_{+}\right)-V\left(M_{-}\right)\right]$are hermitian operators since the representation $V$ is unitary.

An interesting feature of the representation (31) of $K$ is that it is reducible. There exists two closed invariant subspaces of $\mathscr{B}$ namely the set of even and odd functions respectively. We shall denote them by $\mathscr{B}^{+}$and $\mathscr{B}^{-}$:

$$
\mathscr{B} \pm \equiv\{F \in \mathscr{B}, F(-z)= \pm F(z)\}
$$

They both correspond to the same value of the Casimir operator of the Lie algebra of $K$ :

$$
\left\{\begin{array}{l}
\mathscr{C}=-M_{0}^{2}+\frac{1}{2}\left(M_{+} M_{-}+M_{-} M_{+}\right) \\
V(\mathscr{C})=\frac{3}{16} .
\end{array}\right.
$$

We now define a set of basis vectors in $\mathscr{B}^{+}$and $\mathscr{B}^{-}$as follows

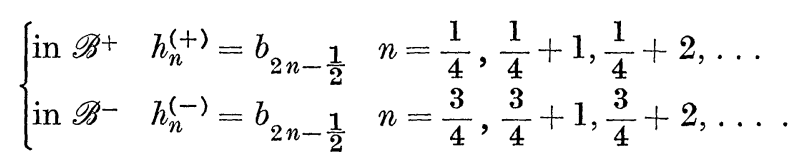

Then in each of these subspaces we have:

$$
\left\{\begin{array}{l}
M^{0} h_{n}^{( \pm)}=n h^{( \pm)} \\
M^{+} h_{n}^{( \pm)}=\sqrt{\frac{3}{16}+n(n+1)} h_{n+1}^{( \pm)} \\
M^{-} h_{n}^{( \pm)}=\sqrt{\frac{3}{16}+n(n-1)} h_{n-1}^{( \pm)}
\end{array}\right.
$$

The formulae look the same, they differ only by the range of $n$. 
These results should be compared to the classification of the (true) unitary representations of $K \equiv S L(2, R)$ given by BARGMANN in reference [3]. They are an extension of the discrete $\operatorname{class}^{1} D_{k}^{+}$to values of $k$ satisfying $\mathscr{C}=k(1-k)=\frac{3}{16}$, i.e. $k=\frac{1}{4}$ and $k=\frac{3}{4}$ in $\mathscr{B}^{+}$and $\mathscr{B}^{-}$ respectively. We shall call them $V^{+}$and $V^{-}$. The following considerations provide a better mean to visualize this analogy.

We want a simpler expression for the representations $V^{ \pm}$. We first consider $V^{+}$and introduce the following matrix element

$$
\lambda^{\frac{1}{2}}\left(b_{0}, V(k) F\right)=\int d \mu(z) F(z) e^{\frac{\bar{\mu}}{2 \lambda} \bar{z}^{2}}=\Phi\left(\frac{\bar{\mu}}{\lambda}\right) .
$$

The function $\Phi$ is obviously an analytic function of its argument inside the unit disk. Hence (39) defines a mapping of $\mathscr{B}$ in the space of analytic functions inside the unit disk. Its kernel is $\mathscr{B}$ - and we restrict immediately this mapping to $\mathscr{B}^{+}$. The case of odd functions will be studied later. If $k$ corresponds to $(\lambda, \mu)$ and $k^{\prime}$ to $\left(\lambda^{\prime}, \mu^{\prime}\right)$, then $k^{\prime \prime}=k k^{\prime}$ corresponds to

$$
\lambda^{\prime \prime}=\lambda^{\prime} \lambda+\bar{\mu} \mu^{\prime} \quad \mu^{\prime \prime}=\bar{\lambda} \mu^{\prime}+\mu \lambda .
$$

The mapping sends $F$ on $\Phi$, it will then send $V\left(k^{\prime}\right) F$ on

$\lambda^{\frac{1}{2}}\left(b_{0}, V(k) V\left(k^{\prime}\right) F\right)=\left(\frac{\lambda}{\lambda^{\prime \prime}}\right)^{\frac{1}{2}} \Phi\left(\frac{\overline{\mu^{\prime \prime}}}{\lambda^{\prime \prime}}\right)=\left(\mu^{\prime} \frac{\bar{\mu}}{\lambda}+\lambda^{\prime}\right)-\frac{1}{2} \Phi\left(\frac{\bar{\lambda}^{\prime} \frac{\bar{\mu}}{\lambda}+\bar{\mu}^{\prime}}{\mu^{\prime} \frac{\bar{\mu}}{\lambda}+\lambda^{\prime}}\right)$.

Using the same notation for the representation $V^{+}$we have then

$$
V^{+}(k) \Phi(s)=(\mu s+\lambda)^{-\frac{1}{2}} \Phi\left(\frac{\lambda s+\bar{\mu}}{\mu s+\lambda}\right) .
$$

The complex variable $s$ is such that $|s|<1$ and it is clear that the transformations

$$
s \rightarrow k^{-1} s=\frac{\bar{\lambda} s+\bar{\mu}}{\mu s+\lambda} \quad k=\left(\begin{array}{cc}
\bar{\lambda} & -\mu \\
-\bar{\mu} & \lambda
\end{array}\right)
$$

are just the usual automorphisms of the unit disk. Note that $\mu s+\lambda$ never vanishes for $|s|<1$.

We shall study the mapping (38) in more detail. The image of $b_{2 m}$ is

$$
\left\{\begin{array}{c}
\Phi_{2 m}(s)=[(2 m) !]^{-\frac{1}{2}} \int d \mu(z) z^{2 m} e^{\frac{s \bar{z}^{2}}{2}} \\
=\frac{[(2 m) !]^{\frac{1}{2}}}{2^{m} m !} s^{m}=\left[\frac{\Gamma\left(m+\frac{1}{2}\right)}{\Gamma(m+1) \Gamma\left(\frac{1}{2}\right)}\right]^{\frac{1}{2}} s^{m} \\
m=0,1,2, \ldots .
\end{array}\right.
$$

1 The reader should not confuse the symbol $k$ used here and only here as the subscript for a representation, with $k$ an arbitrary element in the group $K$. 
Define now a Hilbert space of functions analytic in the unit disk, $\mathscr{H}_{1 / 2}$, in the following way

$\Phi \in \mathscr{H}_{\frac{1}{2}} \Leftrightarrow|s|<1, \quad \Phi(s)=\sum_{m=0}^{\infty} c_{m} \Phi_{2 m}(s), \quad\|\Phi\|^{2}=\sum_{m=0}^{\infty}\left|c_{m}\right|^{2}<\infty$.

Note that the Taylor series of $\Phi$ converges uniformly on any compact subset of the unit disk since

$$
\left\{\begin{array}{l}
|\Phi(s)|^{2} \leqq \sum_{m=0}^{\infty}\left|c_{m}\right|^{2} \sum_{m=0}^{\infty}\left|\Phi_{2 m}(s)\right|^{2}=\|\Phi\|^{2}(1-s \bar{s})^{-\frac{1}{2}} \\
s \bar{s}<1
\end{array}\right.
$$

due to the fact that for

$$
|x|<1,(1-x)^{-\frac{1}{2}}=\sum_{m=0}^{\infty} \frac{\Gamma\left(m+\frac{1}{2}\right)}{\Gamma(m+1) \Gamma\left(\frac{1}{2}\right)} x^{m},
$$

for that branch of the function (which we consider) which is real positive for $x=0$. The inequality (42) tells us also that any Cauchy sequence in $\mathscr{H}_{1 / 2}$ converges to an element of $\mathscr{H}_{1 / 2}$ in the Hilbert space norm and in the usual sense uniformly on any compact subset of the unit disk.

The scalar product in $\mathscr{H}_{1 / 2}$ is defined in an obvious way when the functions are expanded in terms of the complete set $\left\{\Phi_{m}\right\}$ and the mapping (38) is then a unitary map between $\mathscr{B}^{+}$and $\mathscr{H}_{1 / 2}$. Then the formula (39) defines in $\mathscr{H}_{1 / 2}$ a unitary double valued representation of the canonical group $K$ in $\mathscr{H}_{1 / 2}$.

The Hilbert space $\mathscr{H}_{1 / 2}$ has the usual features of a Hilbert space of analytic functions. Apart from the inequality (42) we have a reproducing kernel as an immediate consequence of (38). Indeed since the correspondence $F \rightarrow \Phi$ preserves the scalar product we have

$$
\begin{gathered}
\Phi\left(\frac{\bar{\mu}}{\lambda}\right)=\lambda^{\frac{1}{2}}\left(V\left(k^{-1}\right) b_{0}, F\right)_{\mathscr{B}}=\lambda^{\frac{1}{2}}\left(V\left(k^{-1}\right) \Phi_{0}, \Phi\right)_{\mathscr{H}_{1 / 2}} \\
\Phi_{0}(s)=1, \bar{\lambda}^{\frac{1}{2}}\left[V\left(k^{-1}\right) \Phi_{0}\right](s)=\bar{\lambda}^{\frac{1}{2}}(\bar{\lambda}-\mu s)^{-\frac{1}{2}=\left(1-\frac{\mu_{s}}{\bar{\lambda}}\right)^{-\frac{1}{2}}}
\end{gathered}
$$

So that:

$$
\Phi(s)=\left(\Phi_{s}, \Phi\right)_{\mathscr{H}_{1 / 2}} ; \Phi_{S}\left(s^{\prime}\right)=\left(1-\bar{s} s^{\prime}\right)^{-\frac{1}{2}}\left|s^{\prime}\right|<1 .
$$

And in (43) the square root has a positive real part.

We compute the matrix elements of $V^{+}$. Denote $V_{n, m}^{+}(k)$ the matrix element

$$
V_{n, m}^{+}(k)=\left(\Phi_{2 n}, V^{+}(k) \Phi_{2 m}\right) .
$$


Then a direct computation leads to:

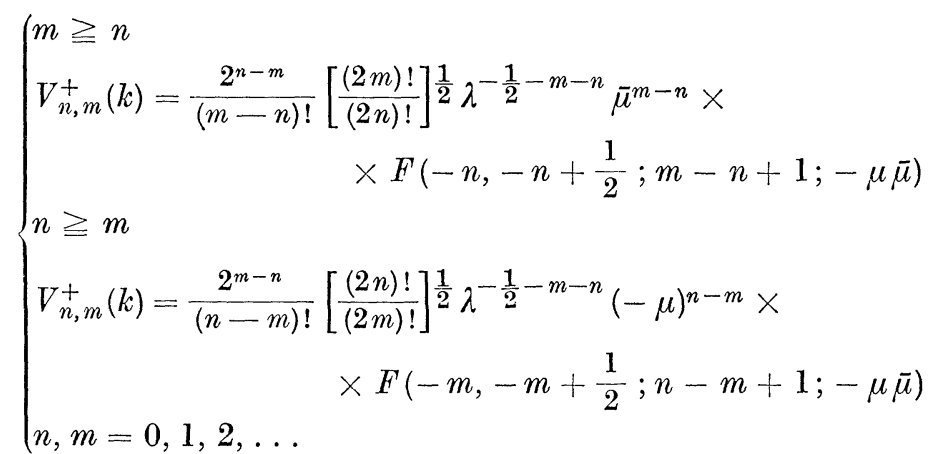

where $F(a, b ; c ; x)$ is the usual hypergeometric function.

We wish to give a similar analysis of the representation $V^{-}$. For that purpose we replace (38) by an other matrix element which has the same virtue, namely except for an extra factor, to depend on $k$ only through the combination $\frac{\bar{\mu}}{\lambda}$. An obvious candidate is:

$$
\lambda^{\frac{3}{2}}\left(b_{1}, V(k) F\right)=\int d \mu(z) F^{\prime}(z) \bar{z} e^{\frac{\bar{\mu}}{\lambda 2} \bar{z}^{2}}=\Psi\left(\frac{\bar{\mu}}{\lambda}\right) .
$$

Again we thus define a mapping $F \rightarrow \Psi$ of $\mathscr{B}$ in the space of analytic functions inside the unit disk, the kernel of which is $\mathscr{B}^{+}$. We restrict ourself to $\mathscr{B}^{-}$and call $\mathscr{H}_{3 / 2}$ the image of $\mathscr{B}^{-}$. $\mathscr{H}_{3 / 2}$ is given a Hilbert space structure by choosing as an orthonormal basis the images $\Psi_{2 m+1}$ of $b_{2 m+1}, m=0 ; 1,2, \ldots$

$$
\left\{\begin{aligned}
& \Psi_{2 m+1}(s)=[(2 m+1) !]^{-\frac{1}{2}} d \mu(z) z^{2 m+1} \bar{z} e^{\frac{s \bar{z}^{2}}{2}}=\frac{[(2 m+1) !]^{\frac{1}{2}}}{2^{m} m !} s^{m} \\
&=\left[\frac{\Gamma\left(m+\frac{3}{2}\right)}{\Gamma(m+1) \Gamma\left(\frac{3}{2}\right)}\right]^{\frac{1}{2}} s^{m} \\
& m=0,1,2, \ldots
\end{aligned}\right.
$$

Then $\mathscr{H}_{3 / 2}$ has the following definition

$\Psi \in \mathscr{H}_{3 / 2} \Leftrightarrow|s|<1, \Psi(s)=\sum_{m=0}^{\infty} c_{m} \Psi_{2 m+1}(s),\|\Psi\|^{2}=\sum_{m=0}^{\infty}\left|c_{m}\right|^{2}<\infty$.

In this case one has also the possibility of defining the scalar product through an integral; indeed:

So that

$$
\frac{1}{2} \int \frac{d^{2} s}{\pi}(1-s \bar{s})^{-\frac{1}{2}} \bar{\Psi}_{2 m_{1}+1}(s) \Psi_{2 m_{2}+1}(s)=\delta_{m_{1} m_{2}} .
$$

$$
\left(\Psi_{1}, \Psi_{2}\right)=\frac{1}{2} \int \frac{d^{2} s}{\pi}(1-s \bar{s})^{-\frac{1}{2} \Psi_{1}(s)} \Psi_{2}(s) .
$$


This could not be achieved for $\mathscr{H}_{1 / 2}$ the corresponding integral being divergent. In complete analogy to what we obtained before we also have:

$$
\left\{\begin{array}{l}
\Psi \in \mathscr{H}_{\frac{3}{2}} \Rightarrow|\Psi(s)|^{2} \leqq\|\Psi\|^{2}(1-s \bar{s})^{-\frac{3}{2}} \\
\Psi(s)=\left(\Psi_{s}, \Psi\right), \quad \Psi_{s}\left(s^{\prime}\right)=\left(1-\bar{s} s^{\prime}\right)^{-\frac{3}{2}}
\end{array}\right.
$$

The representation $V^{-}$of the canonical group $K$ yields in $\mathscr{H}_{3 / 2}$

$$
V^{-}(k) \Psi(s)=(\mu s+\lambda)^{-\frac{3}{2}} \Psi\left(\frac{\bar{\lambda} s+\mu}{\mu s+\lambda}\right)
$$

leading for the matrix elements

$$
V_{n, m}^{-}(k)=\left(\Psi_{2 n+1}, V^{-}(k) \Psi_{2 m+1}\right)
$$

to the following expressions:

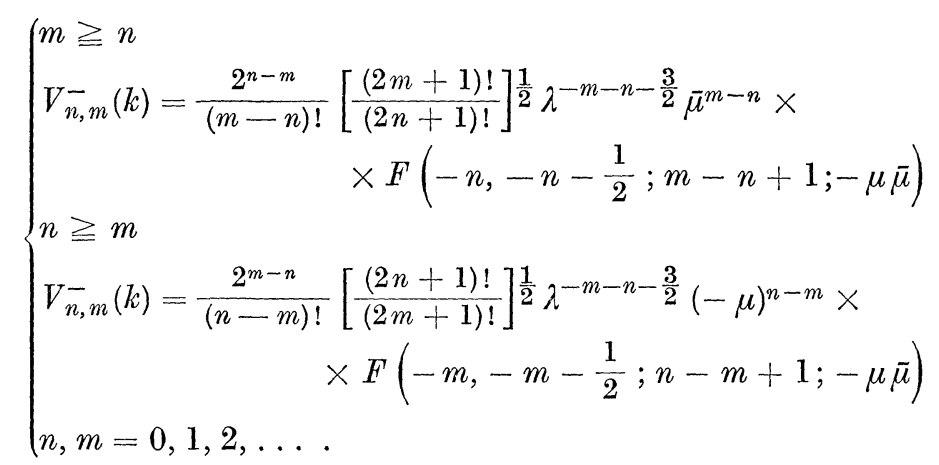

One can observe that $\mathscr{H}_{1 / 2} \subset \mathscr{H}_{3 / 2}$ the inclusion being continuous since for any $\Phi \in \mathscr{H}_{1 / 2}$ one has

$$
\|\Phi\|_{\mathscr{H}_{3 / 2}} \leqq\|\Phi\|_{\mathscr{H}_{1 / 2}}
$$

In the Hilbert space $\mathscr{H}_{1 / 2} \oplus \mathscr{H}_{3 / 2}$ we can write any vector as a set of two functions $\left(\begin{array}{l}\Phi \\ \Psi\end{array}\right)$ with $\Phi \in \mathscr{H}_{1 / 2}, \Psi \in \mathscr{H}_{3 / 2}$. The operators $A$ and $A^{+}$ take the following form

$$
A=\left[\begin{array}{cc}
0 & 1 \\
2 \frac{d}{d s} & 0
\end{array}\right] \quad A^{+}=\left[\begin{array}{cc}
0 & s \\
2 s \frac{d}{d s}+1 & 0
\end{array}\right] \quad\left[A, A^{+}\right]=1 .
$$

The quadratic forms in $A, A^{+}$are diagonal as they should, and do not involve derivatives of order higher than one:

$$
\begin{gathered}
\frac{1}{2}\left(\frac{1}{2}+A^{+} A\right)=\left[\begin{array}{cc}
\frac{1}{4}+s \frac{d}{d s} & 0 \\
0 & \frac{3}{4}+s \frac{d}{d s}
\end{array}\right], \\
\frac{1}{2} A^{+2}=\left[\begin{array}{cc}
s^{2} \frac{d}{d s}+\frac{s}{2} & 0 \\
0 & s^{2} \frac{d}{d s}+\frac{3 s}{2}
\end{array}\right], \quad \frac{1}{2} A^{2}=\left[\begin{array}{cc}
\frac{d}{d s} & 0 \\
0 & \frac{d}{d s}
\end{array}\right] .
\end{gathered}
$$


It is possible to compare this representation of the Lie algebra of $K$ (equations (32) and (33)) with the global form (equations (39) and (51)) and see that they agree.

\section{Generalization to higher dimensions}

We study briefly the case of $N$ degrees of freedom. The Hilbert space $\mathscr{B}_{N}$ is now the set of entire functions of $N$ variables $f(z), z=\left\{z_{1}, \ldots, z_{N}\right\}$, such that

with

$$
\int d \mu_{N}(z)|f(z)|^{2}=\|f\|^{2}<\infty
$$

and

$$
d \mu_{N}(z)=e^{-z \bar{z}} d \operatorname{Re} z_{1} \ldots d \operatorname{Im} z_{N} \pi^{-N}
$$

The monomials

$$
z \cdot \bar{z}=\sum_{1}^{N} z_{i} \cdot \bar{z}_{i}
$$

$$
\frac{z^{[m]}}{\sqrt{[m !]}}=\frac{z_{1}^{m_{1}} \ldots z_{N}^{m_{N}}}{\sqrt{m_{1} ! \ldots m_{N} !}}
$$

form a complete orthonormal basis and the Weyl group is replaced by the group $W_{N}$ defined as follows. An element $g \in W_{N}$ is parametrized by a complex vector $\alpha=\left\{\alpha_{1}, \ldots, \alpha_{N}\right\}$ and a real number $\varrho$ with the group law $g\left(\alpha^{\prime}, \varrho^{\prime}\right) g\left(\alpha^{\prime \prime}, \varrho^{\prime \prime}\right)=g\left(\alpha+\alpha^{\prime}, \varrho+\operatorname{Im} \alpha^{\prime} \cdot \bar{\alpha}^{\prime \prime}\right)$ with $\alpha^{\prime} \cdot \bar{\alpha}^{\prime \prime}=\sum_{1}^{N} \alpha_{i}^{\prime} \bar{\alpha}_{i}^{\prime \prime}$. The group $W_{N}$ is not the product of $N$ groups $W$. The canonical group $K$ is then the set of continuous automorphisms of $W_{N}$ of the type

$$
k: g(\alpha, \varrho) \rightarrow g(\hat{\alpha}, \varrho) \quad \hat{\alpha} \text { function of } \alpha .
$$

The conditions to be satisfied by $k$ are similar to the ones encountered in the previous section leading to the answer

$$
\left\{\begin{array}{l}
\hat{\alpha}_{i}=\sum_{j}\left(\bar{\lambda}_{i j} \alpha_{j}-\mu_{i j} \bar{\alpha}_{j}\right) \\
\delta_{j k}=\sum_{i} \bar{\lambda}_{i j} \lambda_{i k}-\bar{\mu}_{i j} \mu_{i k} \\
0=\sum_{i} \lambda_{i j} \mu_{i k}-\mu_{i j} \lambda_{i k} .
\end{array}\right.
$$

The group $K_{N}$ is isomorphic to the set of complex $2 N \times 2 N$ matrices which we denote by the same symbol

$$
k=\left(\begin{array}{cc}
\bar{\lambda} & -\mu \\
-\bar{\mu} & \lambda
\end{array}\right) \begin{aligned}
& \lambda+\lambda-\mu^{+} \mu=I_{N} \\
& \lambda^{T} \mu-\mu^{T} \lambda=0
\end{aligned}
$$

where $\lambda$ and $\mu$ stand for the $N \times N$ matrices $\left\{\lambda_{i j}\right\}$ and $\left\{\mu_{i j}\right\}$ and for a matrix $\sigma$ the symbols $\bar{\sigma}, \sigma^{T}$ and $\sigma^{+}$mean complex conjugate, transpose 
and hermitian conjugate. The restrictions on $k$ are equivalent to

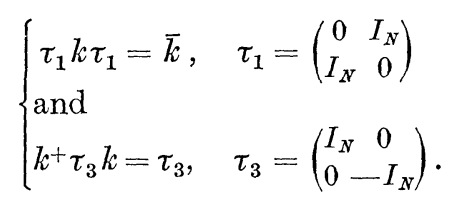

We remark that $k^{-1}$ is obtained by the transformation $\lambda \rightarrow \lambda^{+}, \mu \rightarrow-\mu^{T}$. The group $K_{N}$ is in fact isomorphic to the real symplectic group in $2 N$ dimensions $\operatorname{Sp}(2 N, \mathbb{R})$ (of course $\operatorname{Sp}(2, \mathbb{R}) \approx S L(2, \mathbb{R})$ ). Then for the Lie algebra one has

$$
\sum_{i} \alpha_{i}{ }^{k} A_{i}^{+}-\bar{\alpha}_{i}^{k} A_{i}=\sum_{i j}\left[\left(\bar{\lambda}_{i j} \alpha_{j}-\mu_{i j} \bar{\alpha}_{j}\right) A_{i}^{+}-\left(\lambda_{i j} \bar{\alpha}_{j}-\bar{\mu}_{i j} \alpha_{j}\right) A_{i}\right]
$$

or

$$
{ }^{k} A_{i}^{+}=A_{j}^{+} \bar{\lambda}_{j i}+A_{j} \bar{\mu}_{j i} ; \quad{ }^{k} A_{i}=A_{j} \lambda_{j i}+A_{j}^{+} \mu_{j i}
$$

with $k^{\prime}\left({ }^{k} A\right)={ }^{k k^{\prime}} A$.

The representation of $W_{N}$ in $\mathscr{B}_{N}$ is easily obtained as

$$
U_{[n],[m]}(\alpha, \varrho)=\sqrt{\frac{[m !]}{[n !]}} e^{i \varrho-\frac{\alpha \cdot \bar{\alpha}}{2}} \alpha^{[n-m]} \prod_{1 \leqq i \leqq N} L_{m_{i}}^{n_{i}-m_{i}}\left(\alpha_{i} \bar{\alpha}_{i}\right) .
$$

We now look for operators $V(k)$ with the property that

$$
U\left({ }^{k} g\right)=V(k) U(g) V^{-1}(k) .
$$

The method is the same as before. If $F_{t}(z)=e^{z \cdot \bar{t}-\frac{t \bar{t}}{2}}$ denote as before the reproducing vectors we have to solve the set of equations:

$$
\sum_{j=1}^{N}\left(\lambda_{j i} \frac{\partial}{\partial z_{j}}+\mu_{j i} z_{j}-\bar{t}_{i}\right) V(k) F_{t}(z)=0 \quad i=1, \ldots, N .
$$

Due to the equations (56) the matrix $\lambda$ has an inverse and $\lambda^{-1} \bar{\mu}$ as well as $\mu \lambda^{-1}$ are symmetric. Solving the linear system above, we obtain:

$$
V(k) F_{t}(t)=(\operatorname{det} \lambda)^{-\frac{1}{2}} \exp \left\{-\frac{1}{2} z \mu \lambda^{-1} z+\bar{t} \lambda^{-1} z+\frac{1}{2} \bar{t} \lambda^{-1} \bar{\mu} \bar{t}-\frac{t \bar{t}}{2}\right\} .
$$

In (60) the normalization factor is such that $\left\|V(k) F_{t}\right\|=1$. This leads to the following formula for an arbitrary function $F$ (which is in terms of an absolutely convergent integral)

$V(k) F(t)$

$$
=(\operatorname{det} \lambda)^{-\frac{1}{2}} \int d \mu_{N}(z) \exp \left\{\frac{1}{2} \bar{z} \lambda^{-1} \bar{\mu} \bar{z}+\bar{z} \lambda^{-1} t-\frac{1}{2} t \mu \lambda^{-1} t\right\} F(z) .
$$

One observes on (61) that indeed (60) is verified and further that

(i) $V(k)$ is unitary

(ii) $V(k) U(g) V^{-1}(k)=U\left({ }^{k} g\right)$,

(iii) $V\left(k^{\prime}\right) V(k)= \pm V\left(k^{\prime} k\right)$. 
We shall carry out explicitely the calculation for point (iii)

$V\left(k^{\prime}\right) V(k) F(t)=\left(\operatorname{det} \lambda^{\prime}\right)^{-\frac{1}{2}}(\operatorname{det} \lambda)^{-\frac{1}{2}} \int d \mu_{N}(z) d \mu_{N}\left(z^{\prime}\right) F(z)$

$\exp \left\{\frac{1}{2} \bar{z}^{\prime} \lambda^{\prime-1} \bar{\mu}^{\prime} \bar{z}^{\prime}+\bar{z}^{\prime} \lambda^{\prime-1} t-\frac{1}{2} t \mu^{\prime} \lambda^{\prime-1} t+\frac{1}{2} \bar{z} \lambda^{-1} \bar{\mu} z+\bar{z} \lambda^{-1} z^{\prime}-\frac{1}{2} z^{\prime} \mu \lambda^{-1} z^{\prime}\right\}$

We carry the integration over $z^{\prime}$ with the help of the following consequence of equation (1) valid for $M$ and $N$ symmetric matrices such that $1-\left(\frac{\bar{M}+\bar{N}}{2}\right)\left(\frac{M+N}{2}\right)$ be definite positive (for the proof, see appendice II): $\int d \mu_{N}(z) \exp \left\{+\frac{1}{2} z M z+u z+\frac{1}{2} \bar{z} \bar{N} \bar{z}+\bar{v} \bar{z}\right\}$

$=[\operatorname{det}(1-M \bar{N})]^{-\frac{1}{2}} \times$

$\times \exp \left\{\frac{1}{2}\left[u \bar{N}(1-M \bar{N})^{-1} u+2 \bar{v}(1-M \bar{N})^{-1} u+\bar{v}(1-M \bar{N})^{-1} M \bar{v}\right]\right\}$.

So that with $\lambda^{\prime \prime}=\lambda^{\prime} \lambda+\bar{\mu}^{\prime} \mu$ and $\mu^{\prime \prime}=\bar{\lambda}^{\prime} \mu+\mu^{\prime} \bar{\lambda}$

$V\left(k^{\prime}\right) V(k) F(t)= \pm\left[\operatorname{det} \lambda^{\prime \prime}\right]^{-\frac{1}{2}} \int d \mu_{N}(z) F(z) \times$

$\times \exp \left\{-\frac{1}{2} t \mu^{\prime} \lambda^{\prime-1} t+\right.$

$\left.+\frac{1}{2} \bar{z} \lambda^{-1} \bar{\mu} z+\frac{1}{2} \bar{z} \lambda^{-1} \mu^{\prime}+\lambda^{\prime \prime} T-1 \bar{z}+\bar{z} \lambda^{\prime \prime-1} t-\frac{1}{2} t \lambda^{\prime-1^{T}} \mu \lambda^{\prime \prime-1} t\right\}$.

Now with the help of the definitions of $\lambda^{\prime \prime}$ and $\mu^{\prime \prime}$ and (56) we find

and

$$
\lambda^{-1} \bar{\mu}+\lambda^{-1} \mu^{\prime}+\lambda^{\prime \prime T-1}=\mu^{\prime \prime}+\lambda^{\prime \prime} T-1=\left(\lambda^{\prime \prime-1} \bar{\mu}^{\prime \prime}\right)^{T}
$$

so that:

$$
\mu^{\prime} \lambda^{\prime-1}+\lambda^{\prime-1^{T}} \mu \lambda^{\prime \prime-1}=\mu^{\prime \prime} \lambda^{\prime \prime-1}
$$

$V\left(k^{\prime}\right) V(k) F(t)$

$= \pm\left[\operatorname{det} \lambda^{\prime \prime}\right]^{-\frac{1}{2}} \int d \mu_{N}(z) F(z) \exp \left\{\frac{1}{2} \bar{z} \lambda^{\prime \prime-1} \bar{\mu}^{\prime \prime} \bar{z}+\bar{z} \lambda^{\prime \prime-1} t-\frac{1}{2} t \mu^{\prime \prime} \lambda^{\prime \prime-1} t\right\}$.

Now one checks easily that $k^{\prime \prime}=k^{\prime} k$ has for parameters $\lambda^{\prime \prime}$ and $\mu^{\prime \prime}$ as defined above, hence comparing our result with $V\left(k^{\prime \prime}\right) F(t)$ given by $(61)$ we see that we have indeed proved our statement (iii). In other words we have a projective unitary representation of the canonical group $K_{N} \approx \operatorname{Sp}(2 N, \mathbb{R})$

The representation $V$ is reducible in two irreducible ones acting in the subspaces $\mathscr{B}_{N}^{+}$and $\mathscr{B}_{N}$ of even and odd functions of $\mathscr{B}_{N}$. As in the one dimensional case we can map $\mathscr{B}_{N}^{+}$and $\mathscr{B}_{N}$ on Hilbert spaces of analytic functions defined on an homogeneous space for the group $K_{N}$. Consider first

$(\operatorname{det} \lambda)^{\frac{1}{2}}\left(b_{[0]}, V(k) F\right)=\int d_{N}(z) \exp \left\{\frac{1}{2} \bar{z} \lambda^{-1} \bar{\mu} \bar{z}\right\} F(z)=\Phi\left(\lambda^{-1} \bar{\mu}\right)$.

8 Commun. math. Phys., Vol. 4 
The $N \times N$ matrix $S=\lambda^{-1} \bar{\mu}$ is symmetric and satisfies the condition

$$
S \in \mathscr{S}_{N}
$$

as one readily derives from (56). We shall call $\mathscr{H}_{N}^{1 / 2}$ the image under (63) of the space $\mathscr{B}_{N}^{+}$. It is given a Hilbert space structure by defining an orthonormal basis as the image of $\left\{b_{[m]} ;|m|\right.$ even $\} ;|m|=\sum_{i=1}^{N} m_{i}$. We shall call these functions $\Phi_{[m]}$ :

$$
\begin{gathered}
\Phi_{m}(s)=\int d \mu(z) e+\frac{1}{2} \bar{z} S \bar{z} \frac{z^{[m]}}{\sqrt{[m !]}}, \quad|m| \text { even }=2 p \\
=\frac{\sqrt{[m !]}}{2^{p}} \sum_{\alpha_{i j} \geqq 0} \prod_{i j} \frac{s_{i j}^{\alpha_{i j}}}{\alpha_{i j} !} \\
\sum_{j} \alpha_{i j}+\alpha_{j i}=m_{i} .
\end{gathered}
$$

The function $\Phi_{[m]}$ is an homogeneous polynomial of degree $\frac{|m|}{2}=p$ in the coefficients of the matrix $S$. According to (62)

$$
\sum_{[m]} \frac{t^{[m]}}{\sqrt{[m !]}} \Phi_{[m]}(S)=e^{\frac{t S t}{2}}
$$

if we let $\Phi_{[m]}=0$ for $|m|=2 p+1$.

For a given $|m|=2 p$ the number of functions $\Phi_{[m]}$ is $\left(\begin{array}{c}N+2 p-1 \\ 2 p\end{array}\right)$ while the number of homogeneous polynomials of degree $p$ in the

$$
\frac{N(N+1)}{2} \text { variables } s_{i j} \text { is }\left(\frac{N(N+1)}{2}+p-1\right) \text {. }
$$

These two numbers are unequal in general, except for the case $N=1$ already studied. We shall prove that the functions $\Phi(S)$ that we consider, satisfy a system of partial differential equations for $N \geqq 2$. Indeed consider

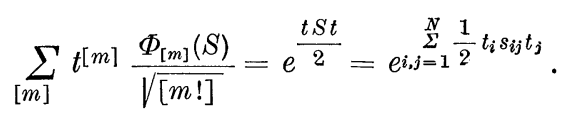

and let $K_{i j}=K_{j i}$ stand for $2^{\delta_{i j}-1} \frac{\partial}{\partial s i j}$ then:

$$
\begin{gathered}
K_{i j} e^{\frac{t S t}{2}}=\frac{1}{2} t_{i} t_{j} e^{\frac{t S t}{2}} \\
\left(K_{i j} K_{l m}-K_{i m} K_{l j}\right) e^{\frac{t S t}{2}}=0 .
\end{gathered}
$$

Since this identity is valid for all values of $t$ we deduce that:

$$
\left\{\begin{array}{l}
\left(K_{i j} K_{l m}-K_{i m} K_{l j}\right) \Phi_{[m]}(S)=0 \\
K_{i j}=2^{\delta_{i j}-1} \frac{\partial}{\partial s_{i j}}=K_{j i}
\end{array}\right.
$$


According to lemma 6 proved in appendix II the necessary and sufficient condition for a polynomial $\Phi(S)$ in the variables $s_{i j}=s_{j i}$ to be written as a linear combination of the $\Phi_{[m]}(S)$ is precisely that

$$
\left(K_{i j} K_{l m}-K_{i m} K_{l j}\right) \Phi(S)=0
$$

for all values of the indices $i, j, l, m$ from 1 to $N$. Moreover, this expansion is unique. We are now in position to give a formal definition of the Hilbert space $\mathscr{H}_{N}^{1 / 2}$. It is the set of functions in the coefficients of the $N \times N$ symmetric matrices $S$, analytic in the generalized unit disk $\mathscr{S}_{N}$ satisfying the second order partial differential equations

$$
\left\{\begin{array}{l}
\left(K_{i j} K_{l m}-K_{i m} K_{l j}\right) \Phi(S)=0 \\
1 \leqq i, j, l, m \leqq N
\end{array}\right.
$$

which insures that for all $S$ subject to the restriction (64) they can be written in a unique way as a power series

$$
\Phi(S)=\sum_{[m]} c_{[m]} \Phi_{[m]}(S)
$$

uniformly convergent in any compact subset of the generalized unit disk; finally one requires that

$$
\|\Phi\|^{2}=\sum_{[m]}\left|c_{[m]}\right|^{2}<\infty .
$$

In this summation only the terms with $|m|$ even enter since if $|m|$ is odd $\Phi_{[m]}=0$. According to the construction above any such $\Phi$ is the image of a unique even function $F$ belonging to $\mathscr{B}_{N}$ through an identity of the type (63). Taking into account the fact that $\|F\|_{\mathscr{B}_{N}}=\|\Phi\|_{\mathscr{H}} \mathscr{C}_{N}^{1 / 2}$ and the unitarity of $V$

$$
\left|\Phi\left(\lambda^{-1} \bar{\mu}\right)\right| \leqq\|\Phi\||\operatorname{det} \lambda|^{\frac{1}{2}}=(\operatorname{det} \lambda+\lambda)^{\frac{1}{4}}\|\Phi\| ;
$$

if $s=\lambda^{-1} \bar{\mu}$ we deduce from $\lambda \lambda^{+}-\bar{\mu} \mu^{T}=I$ that $1-S \bar{S}=\left(\lambda^{+} \lambda\right)^{-1}$ so that:

$$
|\Phi(S)| \leqq\|\Phi\| \operatorname{det}(1-S \bar{S})^{-\frac{1}{4}}
$$

The reproducing kernel $K\left(\bar{S}_{1}, S_{2}\right)=\sum_{[m]} \overline{\Phi_{[m]}\left(S_{1}\right)} \Phi_{[m]}\left(S_{2}\right)$ can also be computed from (63). For that purpose we note that $\mathscr{H}_{N}^{1 / 2}$ is a carrier space of a projective representation $V^{+}$of $K_{N}$ defined by requiring that if $\Phi$ is the image of $F$ then $V\left(k_{1}\right) \Phi$ is the image of $V\left(k_{1}\right) F$ :

$V^{+}\left(k_{1}\right) \Phi\left(\lambda^{-1} \bar{\mu}\right)=(\operatorname{det} \lambda)^{\frac{1}{2}}\left(b_{[0]}, V(k) V\left(k_{1}\right) F\right)= \pm(\operatorname{det} \lambda)^{\frac{1}{2}}\left(b_{[0]}, V\left(k k_{1}\right) F\right)$. Now with

we have

$$
k=\left(\begin{array}{cc}
\bar{\lambda} & -\mu \\
-\bar{\mu} & \lambda
\end{array}\right) \quad k_{1}=\left(\begin{array}{cc}
\bar{\lambda}_{1} & -\mu_{1} \\
-\bar{\mu}_{1} & \lambda_{1}
\end{array}\right)
$$

$$
\begin{gathered}
k_{2}=k k_{1}=\left(\begin{array}{cc}
\frac{\left(\overline{\left(\lambda \lambda_{1}+\bar{\mu} \mu_{1}\right)}-\left(\bar{\lambda} \mu_{1}+\mu \lambda_{1}\right)\right.}{-\left(\bar{\lambda} \mu_{1}+\mu \lambda_{1}\right)} & \lambda \lambda_{1}+\bar{\mu} \mu_{1}
\end{array}\right)=\left(\begin{array}{cc}
\bar{\lambda}_{2} & -\mu_{2} \\
-\bar{\mu}_{2} & \lambda_{2}
\end{array}\right) \\
\lambda_{2}^{-1} \bar{\mu}_{2}=\left(\lambda \lambda_{1}+\bar{\mu} \mu_{1}\right)^{-1}\left(\lambda \bar{\mu}_{1}+\bar{\mu} \bar{\lambda}_{1}\right)=\left[\begin{array}{ll}
\left.\lambda_{1}+\left(\lambda^{-1} \bar{\mu}\right) \mu_{1}\right]^{-1}\left[\bar{\mu}_{1}+\left(\lambda^{-1} \bar{\mu}\right) \bar{\lambda}_{1}\right]
\end{array}\right.
\end{gathered}
$$


and

$$
\begin{gathered}
\operatorname{det}\left(\lambda^{-1} \lambda_{2}\right)=\operatorname{det}\left(\lambda_{1}+\left(\lambda^{-1} \bar{\mu}\right) \mu_{1}\right) \\
{\left[V^{+}(k) \Phi\right](S)=\operatorname{det}(\lambda+S \mu)^{-\frac{1}{2}} \Phi\left((\lambda+S \mu)^{-1}(\bar{\mu}+S \bar{\lambda})\right) .}
\end{gathered}
$$

The transformations $S \rightarrow(\lambda+S \mu)^{-1}(\bar{\mu}+S \bar{\lambda})$ with $\lambda, \mu$ satisfying the conditions (56) are the well known automorphisms of the generalized unit disk. The double valuedness of the representation $V^{+}$is included in the root of the determinant. That the latter never vanishes for $S$ in the generalized unit disk stems from $\operatorname{det}(\lambda+S \mu)=\operatorname{det} \lambda \operatorname{det}\left(I+S \mu \lambda^{-1}\right)$, now $\operatorname{det} \lambda \neq 0$ and $\mu \lambda^{-1}$ is symmetric and also belongs to the generalized unit disk. We can then apply lemma 3 of appendix II which gives $\operatorname{det}\left(I+S \mu \lambda^{-1}\right) \neq 0$.

The reproducing kernel easily follows; we have again

$$
\Phi\left(\lambda^{-1} \bar{\mu}\right)=(\operatorname{det} \lambda)^{\frac{1}{2}}\left(b_{[0]}, V(k) F\right)_{\mathscr{B}_{N}}=\left((\operatorname{det} \bar{\lambda})^{\frac{1}{2}} V\left(k^{-1}\right) \Phi_{[0]}, \Phi\right)_{\mathscr{H}_{N}^{1 / 2}}
$$

since $\Phi_{[0]}$ is the image of $b_{[0]}$. We have already observed that $k^{-1}$ is deduced from $k$ by substituting $\lambda^{+}$for $\lambda$ and $-\mu^{T}$ for $\mu$. According to (70) we have:

$$
\begin{aligned}
V\left(k^{-1}\right) \Phi_{[0]}(S) & =\operatorname{det}\left(\lambda^{+}-S \mu^{T}\right)^{-\frac{1}{2}}=\operatorname{det}(\bar{\lambda}-\mu S)^{-\frac{1}{2}} \\
& =(\operatorname{det} \bar{\lambda})^{-\frac{1}{2}}\left(\operatorname{det}\left(I-\overline{\left(\lambda^{-1} \bar{\mu}\right)} S\right)\right)^{-\frac{1}{2}} .
\end{aligned}
$$

So that the reproducing kernel is:

$$
K\left(\bar{S}_{1}, S_{2}\right)=\operatorname{det}\left(1-\bar{S}_{1} S_{2}\right)^{-\frac{1}{2}}=\sum_{[m]} \overline{\Phi_{[m]}\left(S_{1}\right)} \Phi_{[m]}\left(S_{2}\right) .
$$

The phase in (71) is obtained by analytic continuation from $K(0,0)=1$.

One can observe that the transformations (70) are such that the equations (67) are still fullfilled. This is obvious from our construction but can be verified directly (see lemma 7 of appendix II). One also notes that $K_{N}$ contains as a subgroup $U_{N}$ the unitary group in $N$ dimensions, then $V^{+}$restricted to $U_{N}$ is reducible and yields all the representations of the type $g \in U_{N} \rightarrow \operatorname{det} g^{-1 / 2} \times$ a totally symmetric representation of even degree.

The case of odd functions in $\mathscr{B}_{N}^{-}$is a little more involved. To any element in $\mathscr{B}_{N}{ }_{N}$ we associate a vector in an $N$-dimensional space whose components are analytic functions in $\mathscr{S}_{N}$ in the following manner. Denote by $b_{i} 1 \leqq i \leqq N$ the elements $b_{[0,0, \ldots 1,0,0]}=z_{i}$ in $\mathscr{B}_{N}$, we write for an arbitrary $F \in \mathscr{B}_{N}$

$$
(\operatorname{det} \lambda)^{\frac{1}{2}} \sum_{j=1}^{N}\left(b_{j}, V(k) F\right) \lambda_{j i}=\int d \mu_{N}(z) \exp \left\{\bar{z} \lambda^{-1} \bar{\mu} \bar{z}\right\} \bar{z}_{i} F(z)=\Psi_{i}\left(\lambda^{-1} \bar{\mu}\right) .
$$

It is clear that the $N$ functions $\Psi_{i}(S)$ associated to $F$ are analytic in 
$\mathscr{S}_{N}$ and satisfy the same equations (67) as before. We shall call $\mathscr{H}_{N}^{3 / 2}$ the image of $\mathscr{B}_{N}^{-}$. The basic vectors $b_{[m]},|m|$ odd, map on the vectors

$$
\Psi_{[m], i}(S)=\sqrt{m_{i}} \Phi_{\left[m_{1}, \ldots, m_{i}-1, \ldots, m_{N}\right]}(S)
$$

where the $\Phi_{[m]}$ are as before (it is understood that if $m_{i}=0$ then $\Psi_{[m]}$, $i$ is zero). Any $\boldsymbol{\Psi}$ belonging to $\mathscr{H}_{N}^{3 / 2}$ is assumed to be such that

$$
\boldsymbol{\Psi}=\sum_{|m| \text { odd }} d_{[m]} \boldsymbol{\Psi}_{[m]}, \sum\left|d_{[m]}\right|^{2}<\infty .
$$

Clearly the conditions (67) are insufficient to insure that an analytic vector function in the neighborhood of $S=0$ will have an expansion of the type (74). One must add further restrictions relating the various components of $\boldsymbol{\Psi}$. To see this observe that one has:

$$
\sum_{[m]} \frac{t^{[m]}}{\sqrt{[m !]}} \Psi_{[m], i}(S)=t_{i} e^{\frac{t S t}{2}},
$$

from which follows that all the $\boldsymbol{\Psi}_{[m]}$ and hence all the functions $\boldsymbol{\Psi}$ that we consider satisfy the system of equations

$$
K_{i j} \boldsymbol{\Psi}_{l}(S)=K_{i l} \boldsymbol{\Psi}_{j}(S) .
$$

It is clear that in fact for each component of $\boldsymbol{\Psi}$ the equations (67) follow from (75). A slight extension of lemma 6 (appendix II) shows that conversely any vector valued polynomial in the $S_{i j}$ satisfying (75) can be expanded in terms of the $\boldsymbol{\Psi}_{[m]}$.

Schwarz inequality applied to (72) easily yields for any $\Psi(S) \in \mathscr{H}_{N}^{3 / 2}$

$$
\sum_{i=1}^{N} \bar{\Psi}_{i}(S) \Psi_{i}(S) \leqq\|\boldsymbol{\Psi}\|^{2} \operatorname{det}(1-S \bar{S})^{-\frac{1}{2}} \operatorname{Trace}\left[(1-S \bar{S})^{-1}\right], S \in \mathscr{S}_{N}
$$

The representation $V^{-}(k)$ is also derived from the same formula

$$
\begin{gathered}
{\left[V^{-}(k) \Psi\right]_{i}(S)=[\operatorname{det}(\lambda+S \mu)]^{-\frac{1}{2}} \sum_{j} \Psi_{j}\left((\lambda+S \mu)^{-1}(\bar{\mu}+S \bar{\lambda})\right) \times} \\
\times[\lambda+S \mu]_{i j}^{-1} .
\end{gathered}
$$

And finally the reproducing kernel is a set of $N$ elements in $\mathscr{H}_{N}^{3 / 2}$ depending on $S^{\prime}$ such that:

$$
\begin{aligned}
\Psi_{l}\left(S^{\prime}\right) & =\left(K^{l, s^{\prime}}, \Psi\right) \\
K_{i}^{l, s^{\prime}}(S) & =\left[1-S \bar{S}^{\prime}\right]_{l i}^{-1}\left[\operatorname{det}\left(1-S \bar{S}^{\prime}\right)\right]^{-\frac{1}{2}} .
\end{aligned}
$$

In conclusion let us note that restricted to the unitary group $U_{N} \subset K_{N}$ the representation $V$-is equal to the direct sum of all the representations of the form $g \in U_{N} \rightarrow \operatorname{det} g^{-1 / 2} \times$ a totally symmetric representation of odd degree. 


\section{Appendix I}

\section{Laguerre polynomials as matrix elements}

We want to use formula (26) of section II to deduce some classical properties of Laguerre polynomials.

From Schwarz inequality one has $\left|U_{n, m}(\alpha, \varrho)\right| \leqq 1$.

This yields

$$
\left.\left|e^{-\frac{x}{2}}\right| x\right|^{\frac{k}{2}} L_{p}^{k}(x) \mid \leqq\left[\frac{(p+k) !}{p !}\right]^{\frac{1}{2}} p+k \text { positive } .
$$

From the integral representation

$$
\alpha^{k} L_{p}^{k}(\alpha \bar{\alpha})=\int \frac{d^{2} z}{\pi} e^{-z \bar{z}} \frac{\bar{z}^{p+k}(z-\bar{\alpha}) p}{p !} e^{\alpha z}
$$

one can get a generating function since for $|t|<1, k \geqq 0$

$$
\begin{gathered}
(\alpha t)^{k} \sum_{0}^{\infty} t^{p} L_{p}^{k}(\alpha \bar{\alpha})=(-1)^{k}\left(\frac{\partial}{\partial \bar{\alpha}}\right)^{k} \int \frac{d^{2} z}{\pi} e^{-(1-t) z \bar{z}+\alpha z-t \bar{\alpha} \bar{z}} \\
=(-1)^{k}\left(\frac{\partial}{\partial \bar{\alpha}}\right) \frac{1}{1-t} \cdot e^{-t \frac{\alpha \bar{\alpha}}{1-t}} .
\end{gathered}
$$

Hence:

$$
(1-t)^{-k-1} e^{-\frac{t x}{1-t}}=\sum_{0}^{\infty} t^{p} L_{p}^{k}(x)|t|<1, k \geqq 0 .
$$

For $U_{n, m}$ we can use an alternative expression

$$
U_{n, m}(\alpha, 0)=\left\langle n\left|e^{\alpha A^{+}-\bar{\alpha} A}\right| m\right\rangle=\frac{1}{\sqrt{n ! m !}}\left\langle 0\left|A^{n} e^{\alpha A^{+}-\bar{\alpha} A} A^{+^{m}}\right| 0\right\rangle,
$$

where $|0\rangle$ stands for the function $b_{0}(z)=1$. Now $\left[A, A^{+}\right]$commutes with $A$ and $A^{+}$and we can make use of the identity $e^{A+B}=e^{A} e^{B} e^{-\frac{1}{2}[A, B}$. valid for the case $[A[A, B]]=[B[A, B]]=0$. This allows one to write

$$
\alpha^{k} L_{p}^{k}(\alpha \bar{\alpha})=\frac{1}{p !} e^{\alpha \bar{\alpha}} \frac{\partial^{p+\bar{k}}}{\partial(-\bar{\alpha})^{p+k}} \frac{\partial^{m}}{\partial \alpha^{m}} e^{\bar{\alpha} \alpha}, k \geqq 0,
$$

which is equivalent to

$$
L_{p}^{0}(x)=\frac{1}{p !} e^{x} \frac{d^{p}}{d x^{p}} e^{-x} x^{p}, L_{p}^{k}(x)=\frac{(-1)^{k}}{(p+k) !} \frac{d^{k}}{d x^{k}} L_{p+k}^{0}(x) .
$$

From (25) we get:

$$
e^{-x z}(z+1)^{m}=\sum_{n=0}^{m} z^{n} L_{n}^{m-n}(x) .
$$

If we use the fact that in the space $\mathscr{B}, z$ and $\frac{\partial}{\partial z}$ are hermitian conjugate we can cast (80) in the form

$$
\alpha^{k} L_{p}^{k}(\alpha \bar{\alpha})=\frac{1}{p !} \int \frac{d^{2} z}{\pi} e^{-z \bar{z}} \bar{z}^{p+k} z^{p} e^{\alpha z-\bar{\alpha} \bar{z}} .
$$


Let in this integral $z=\sqrt{t e^{i} \Phi}$ and $\alpha$ be real $\alpha^{2}=x>0$. Then:

$$
x^{\frac{k}{2}} L_{p}^{k}(x)=\frac{e^{x}}{p !} \int_{0}^{\infty} d t e^{-t} t^{p+\frac{k}{2}} \int_{0}^{2 \pi} \frac{d \Phi}{2 \pi} e^{-i k \Phi+2 i \sqrt{t} x \sin \Phi},
$$

which also reads

$$
L_{p}^{k}(x)=\frac{e^{x} x^{-\frac{k}{2}}}{p !} \int_{0}^{\infty} d t e^{-t} t^{p+\frac{k}{2}} J_{k}(2 \sqrt{t x})
$$

a classical expression for $L_{p}^{k}$.

Finally we can also express $U_{n, m}(\alpha)$ in the initial Hilbert space $\mathscr{L}^{2}(\mathbb{R})$. Then using (10) written for $\alpha=i x$ one derives:

$$
\begin{aligned}
& \sqrt{\pi} 2^{\frac{n+m}{2}} m !(i x)^{n-m} e^{\frac{x^{2}}{x}} L_{m}^{n-m}\left(x^{2}\right) \\
= & \int_{-\infty}^{+\infty} d w e^{-w^{2}+i \sqrt{2 w x}} H_{n}(w) H_{m}(w) .
\end{aligned}
$$

\section{Appendix II}

\section{Lemmas on symmetric matrices}

We establish a number of results used in the text which have all to do with symmetric matrices. The first of them are "polar decompositions".

Lemma 1. Any symmetric $N \times N$ matrix $S$ can be written:

$$
S=U^{T} D U
$$

with $U$ unitary, $D$ real diagonal with non negative elements.

Proof (i) Let $Q$ be unitary and symmetric. It can be written $e^{i H}$ with $H$ hermitian and symmetric, hence real. Let $Q^{\prime}=e^{\frac{i H}{2}}$ then $Q=Q^{\prime 2}$ $=Q^{\prime T} Q^{\prime}$ since $Q^{\prime}$ is also unitary and symmetric. Thus (85) holds for the symmetric unitary matrices.

(ii) For an arbitrary symmetric $S$ consider the hermitian non negative matrix $\bar{S} S$; let $V^{(0)} \ldots V^{(r)}$ be the orthogonal proper subspaces of the corresponding operator in an $N$ dimensional vector space

$$
V=V^{(0)} \perp V^{(1)} \ldots \perp V^{(r)} .
$$

Restricted to $V^{(k)}, \bar{S} S$ is proportional to unity, the proportionality factor being the $k$-th eigenvalue of $\bar{S} S$. We assume for instance that $0 \leqq \lambda_{0}<\cdots<\lambda_{r}$.

(iii) Let $u$ be a vector belonging to $V^{(k)}, \lambda$ the corresponding eigenvalue of $\bar{S} S$ (for brevity we omit the subscript $k$ ):

$$
\bar{S} S u=\lambda u \quad \lambda \geqq 0 .
$$

We write $S u=v, \bar{S} v=\lambda u$ then $S \bar{v}=\lambda \bar{u}$ and $\bar{S} S \bar{v}=\lambda \bar{S} \bar{u}=\lambda \bar{v}$. Hence $u$ and $\bar{v}$ both belong to the subspace $V^{(k)}$. Choose a basis $u^{(\alpha)}$ in $V^{(k)}$. Write $v^{(\alpha)}=S u^{(\alpha)}$. According to what we have seen $v^{(\alpha)}=\sum_{\beta} t_{\alpha \beta} \bar{u}^{(\beta)}$. 
If the basis is orthonormal (i.e. $\bar{u}^{(\alpha)} u^{(\beta)}=\delta_{\alpha \beta}$ ) we have:

$$
u^{(\beta)} v^{(\alpha)}=u^{(\beta)} S u^{(\alpha)}=t_{\alpha \beta} \text {. }
$$

Since $S$ is symmetric $t_{\alpha \beta}$ is equal to $t_{\beta \alpha}$. Moreover:

$$
\lambda u^{(\alpha)}=\bar{S} S u^{(\alpha)}=\sum_{\beta} t_{\alpha \beta} \bar{S} \bar{u}^{(\beta)}=\sum_{\beta, \gamma} t_{\alpha \beta} t_{\beta \gamma} u^{(\gamma)} .
$$

The symmetric matrix $t=\left\{t_{\alpha \beta}\right\}$ is then such that $t t^{+}=\lambda I$.

If $\lambda$ is zero $t$ is equal to zero (it is sufficient for that to observe that $\left.0=\operatorname{tr} t t^{+}=\sum_{i, j}\left|t_{i j}\right|^{2}\right)$ hence $S V^{(0)}=0$.

If $\lambda \neq 0 Q^{\prime}=\frac{t}{\sqrt{\lambda}}$ is unitary and symmetric; according to (i) it follows that there exists $Q$ unitary such that $t=\sqrt{\lambda} Q^{-1} Q^{-1 T}$. We then construct

$$
w^{(\alpha)}=\sum_{\beta} q_{\alpha \beta} u^{(\beta)}
$$

which gives an other orthonormal basis in $V^{(k)}$ with the property

$$
S w^{(\alpha)}=\sum_{\beta \gamma} q_{\alpha \beta} t_{\beta \gamma} \bar{u}^{(\gamma)}=\sqrt{\lambda} \sum_{\gamma} \bar{q}_{\alpha \gamma} \bar{u}^{(\gamma)}=\sqrt{\bar{\lambda}} \bar{w}^{(\alpha)} .
$$

(In $V^{(0)}$ the $w^{(\alpha)}$, are any orthonormal basis vectors.)

(iv) We conclude from (iii) that there exists an orthonormal basis $w^{(a)}(1 \leqq a \leqq N)$ such that:

$$
S w^{(a)}=\mu_{a} \bar{w}^{(a)}
$$

the $\mu_{a}$ being the roots of the non negative eigenvalues of $\bar{S} S$ each repeated a number of times equal to its multiplicity. Let $D$ be the diagonal matrix

$$
D=\left(\begin{array}{lll}
\mu_{1} \cdot \ddots & & 0 \\
0 & \ddots & \mu_{N}
\end{array}\right)
$$

and $U^{-1}$ the unitary matrix whose $a$-th column is the vector $w^{(a)}$, then the preceding set of equalities is equivalent to:

$$
S U^{-1}=\overline{U^{-1}} D=U^{T} D
$$

or:

$$
S=U^{T} D U .
$$

Note that the square of the diagonal elements of $D$ are the eigenvalues of $\bar{S} S$ or $S \bar{S}$.

Lemma 2. Any element $k$ of the symplectic group $K_{N}$ can be written in the form

$k=g_{1} d g_{2}$
with $g_{1}$ and $g_{2}$ of the form $\left(\begin{array}{cc}\bar{U} & 0 \\ 0 & U\end{array}\right), U: N$ by $N$ unitary matrix, and $d$ of the type:

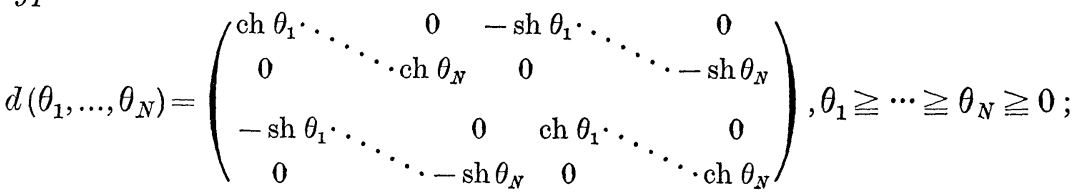

of course $g_{1}, g_{2}$ and $d$ belong to $K_{N}$. 
The proof of this lemma is omitted since it can be found for instance in $[4]$.

Lemma 3. If the symmetric matrices $M$ and $N$ satisfy $1-\bar{M} M>0$ and $1-\bar{N} N>0$ then $\operatorname{det}(1-M N) \neq 0$.

Proof. For any non zero vector $z, \bar{z} \cdot z-\bar{z} \bar{M} M z>0$ and $\bar{z} \cdot z-$ $-\bar{z} \bar{N} N z>0$. Assume that $\operatorname{det}(1-M N)=0$ then there would exist a non zero vector $z_{1}$ such that $z_{1}=M N z_{1}$. Clearly $N z_{1} \neq 0$. Replace in the positivity condition of $M$ the arbitrary $z$ by $N z_{1}$, it reads $\bar{z}, \bar{N} N z_{1}-$ $-\bar{z}_{1} z_{1}>0$ contrary to the hypothesis on $N$.

The next two lemmas have to do with the integration of exponentials of quadratic forms.

Lemma 4. The necessary and sufficient condition for the entire function

$$
e^{\frac{1}{2} z S z}=e^{\frac{1}{2}} \sum_{i, j=1}^{N} z_{i} s_{i j} z_{j}
$$

to belong to the space $\mathscr{B}_{N}$ is that the hermitian matrix

be positive definite.

$$
I-\bar{S} S
$$

Proof. According to equation (1) in the introduction the condition is equivalent to the requirement that:

$$
z \bar{z}-\frac{1}{2} z S z-\frac{1}{2} \bar{z} \bar{S} \bar{z}
$$

be positive for any complex $N$-vector $z$. Using lemma 1 this condition is equivalent to the positivity of:

$$
\sum_{i}\left(z_{i} \bar{z}_{i}-\frac{1}{2} d_{i} z_{i}^{2}-\frac{1}{2} d_{i} \bar{z}_{i}^{2}\right),
$$

where $d_{i}$ are the diagonal elements of $D$ when $S$ is written $U^{T} D U$. Writing $z_{j}=x_{j}+i y_{j}$ we must have:

or

$$
\sum_{i}\left(\left(1-d_{i}\right) x_{i}^{2}+\left(1+d_{i}\right) y_{i}^{2}\right)>0
$$

$$
d_{i}^{2}<1 .
$$

The $d_{i}^{2}$ are precisely the eigenvalues of $\bar{S} S$, hence $1-d_{i}^{2}$ are the eigenvalues of $I-\bar{S} S$ and the conditions we have found are just the statement of the lemma. We remark that the norm of $f(z)=e^{\frac{1}{2} z S z}$ is equal to

$$
\|f\|^{2}=\int d \mu_{N}(z) \overline{f(z)} f(z)=[\operatorname{det}(I-S \bar{S})]^{-\frac{1}{2}} .
$$

Lemma 5. Given two symmetric matrices $M$ and $N$ and two vectors $u$ and $v$ the integral

$$
\int d \mu_{P}(z) \exp \left\{\frac{1}{2}[z M z+2 u \cdot z+\bar{z} \bar{N} \bar{z}+2 \bar{v} \cdot \bar{z}]\right\}
$$


is absolutely convergent if and only if:

$$
I-\left(\frac{\bar{M}+\bar{N}}{2}\right)\left(\frac{M+N}{2}\right)>0
$$

in which case it is equal to

$[\operatorname{det}(I-M \bar{N})]^{-\frac{1}{2}} \times$

$\times \exp \left\{\frac{1}{2}\left[u \bar{N}(1-M \bar{N})^{-1} u+2 \bar{v}(1-M \bar{N})^{-1} u+\bar{v}(1-M \bar{N})^{-1} M \bar{v}\right]\right\}$

the sign of the determinant being obtained through analytic continuation between $0 \leqq \alpha \leqq 1$ from $\left[\operatorname{det}\left(I-M_{\alpha} \bar{N}_{\alpha}\right)\right]^{-1 / 2}$ with:

$$
M_{\alpha}=M\left(\frac{1+\alpha}{2}\right)+N\left(\frac{1-\alpha}{2}\right), \quad N_{\alpha}=N\left(\frac{1+\alpha}{2}\right)+M\left(\frac{1-\alpha}{2}\right)
$$

and

$$
\left[\operatorname{det}\left(1-M_{0} \bar{N}_{0}\right)\right]^{-\frac{1}{2}}=\left[\operatorname{det}\left(1-\frac{M+N}{2} \cdot \frac{\bar{M}+\bar{N}}{2}\right)\right]^{-\frac{1}{2}}>0 .
$$

Proof. First the condition for absolute convergence is obtained by a slight modification of the argument of lemma 4 . It includes the case where $I-\bar{M} M>0$ and $I-\bar{M} N>0$ since these conditions are equivalent to

hence

$$
\begin{aligned}
& z \cdot \bar{z}+\frac{1}{2}(z M z+\bar{z} \bar{M} \bar{z})>0 \\
& z \cdot \bar{z}+\frac{1}{2}(z N z+\bar{z} \bar{N} \bar{z})>0
\end{aligned}
$$

$$
z \cdot \bar{z}+\frac{1}{2}\left(z \frac{M+N}{2} z+\bar{z} \frac{\bar{M}+\bar{N}}{2} \bar{z}\right)>0
$$

in turn equivalent to $\left[I-\left(\frac{\bar{M}+\bar{N}}{2}\right)\left(\frac{M+N}{2}\right)\right]>0$.

To get the result of the integration we apply formula (1); write $z=x+i y$ the integral reads:

$$
\int_{R^{2}} \frac{d^{P} x d^{P} y}{\pi^{P}} e^{-(x, y) A(x, y)+2\left(\frac{u+\bar{v}}{2}, i \frac{u-\bar{v}}{2}\right) \cdot(x, y)},
$$

with $A$ a matrix in the $(x, y)$ space equal to $1 / 2 V^{T} A^{\prime} V$

$$
V=\left(\begin{array}{cc}
I & i \\
I & -i
\end{array}\right), \quad A^{\prime}=\left(\begin{array}{cc}
-M & I \\
I & -\bar{N}
\end{array}\right) .
$$

We have to compute $\operatorname{det} A$ and $A^{-1}$, since

$$
\operatorname{det}\left(\frac{1}{2} V V^{T}\right)=(-1)^{P}, \quad \operatorname{det} A=(-1)^{P} \operatorname{det} A^{\prime}
$$

and with

$$
\sigma=\left(\begin{array}{cc}
0 & -I \\
I & 0
\end{array}\right), \quad A^{\prime} \sigma A^{\prime} \sigma=\left(\begin{array}{cc}
I-M \bar{N} & 0 \\
0 & I-\bar{N} M
\end{array}\right)
$$


hence $\left(\operatorname{det} A^{\prime}\right)^{2} \operatorname{det} \sigma^{2}=(\operatorname{det} I-M \bar{N})^{2}$ since $I-\bar{N} M$ is the transpose of $I-M \bar{N}$. Clearly $\operatorname{det} \sigma=1$ so that

$$
\operatorname{det} A^{\prime}=(-1)^{P} \operatorname{det}(I-M \bar{N}) \text { and } \operatorname{det} A=(I-M \bar{N}),
$$

the sign of $\operatorname{det} A^{\prime}$ being determined by comparing the values of $\operatorname{det} A^{\prime}$ and $\operatorname{det}(I-M \bar{N})$ at a particular point. The above calculation also yields

So that $A^{-1}=2 V^{-1} A^{\prime-1} V^{T^{-1}}$

$$
A^{\prime-1}=\left(\begin{array}{lr}
\bar{N}(I-M \bar{N})^{-1} & (I-\bar{N} M)^{-1} \\
(I-M \bar{N})^{-1} & M(I-\bar{N} M)^{-1}
\end{array}\right) .
$$

$$
V^{T-1}=\frac{1}{2}\left(\begin{array}{cc}
1 & -i \\
1 & i
\end{array}\right), \quad V^{T-1}\left(\begin{array}{c}
\frac{u-\bar{v}}{2} \\
i \frac{u+\bar{v}}{2}
\end{array}\right)=\frac{1}{2}\left(\begin{array}{c}
u \\
\bar{v}
\end{array}\right)
$$

and

$$
\begin{aligned}
& \left(\frac{u+\bar{v}}{2}, i \frac{u-\bar{v}}{2}\right) A^{-1}\left(\begin{array}{c}
\frac{u+\bar{v}}{2} \\
i \frac{u-\bar{v}}{2}
\end{array}\right) \\
& \quad=\frac{1}{2}\left[u \bar{N}(I-M \bar{N}) u+2 \bar{v}(I-M \bar{N})^{-1} u+\bar{v} \cdot(I-M \bar{N})^{-1} \bar{v}\right] .
\end{aligned}
$$

Collecting all these results in formula (1) we obtain the desired result except the sign in front of $\operatorname{det}(I-M \bar{N})^{-1 / 2}$.

For the latter we follow the procedure indicated in the introduction.

Lemma 6. The necessary and sufficient condition for a polynomial $\Phi(S)$ in the coefficients $s_{i j}$ of a symmetric matrix $S$ to be expanded as a linear combination of the $\Phi_{[m]}(s)$ is that $\Phi$ satisfies the partial differential equations

$$
\begin{gathered}
\left(K_{i j} K_{l m}-K_{i m} K_{l j} \Phi(S)=0\right. \\
K_{i j}=\frac{1}{2} \frac{\partial}{\partial S_{i j}} \quad \text { if } \quad i \neq j, K_{i i}=\frac{\partial}{\partial S_{i i}} .
\end{gathered}
$$

The functions $\Phi_{[m]}$ are given by (65). The expansion of $\Phi$ is then unique.

Proof. The necessity proof has been given in the text. Suppose now that the polynomial $\Phi$ satisfies the above equations. It is convenient to recast the expression of $\Phi_{[m]}$ in the following form:

$$
\begin{gathered}
\frac{\frac{2}{|m|}^{2}}{\sqrt{[m !]}} \Phi_{[m]}(S)=\sum_{\alpha_{i} \geqq 0, \alpha_{l m} \geqq 0} \prod_{i, l, m l<m} \frac{S_{i i}^{\alpha_{i}}}{\alpha_{i} !} \frac{\left(2 S_{l m}\right) \alpha_{l m}}{\alpha_{l m} !} . \\
2 \alpha_{i}+\sum_{j<i} \alpha_{j i}+\sum_{l>i} \alpha_{i l}=m_{i} .
\end{gathered}
$$

For each term in $\Phi_{[m]}$ we can arrange the exponents in a symmetric matrix of non negative integers

$$
(\alpha) \equiv\left[\begin{array}{ccc}
2 \alpha_{1} & \alpha_{12} & \cdots \\
\alpha_{21} & 2 \alpha_{2} & \cdots \\
\cdot & . & . \\
. & . & .
\end{array}\right] \alpha_{i j} \equiv \alpha_{j i}
$$


the sum of the elements in the $i$-th row being equal to $m_{i}$. Clearly the sum of all elements equals $\sum_{i} m_{i}$ and is even since each off diagonal element is repeated twice and the diagonal elements are even.

Our polynomial $\Phi$ can also be written

$$
\Phi(S)=\sum_{(\beta)} \varrho(\beta) \prod_{i, l<m} \frac{S_{i i}^{\beta_{i}}}{\beta_{i} !} \frac{\left(2 S_{l m}\right)^{\beta_{l m}}}{\beta_{l m} !}
$$

where $(\beta)$ indicates the exponents written in a similar matrix form as above. The differential equations satisfied by $\Phi$ can be broken in three types according to the number of unequal indices among $i j l m$, namely 2,3 or 4 , leading to three types of equalities for the coefficients $\varrho(\beta)$ namely

$$
\varrho(\beta)=\varrho_{\left(\beta^{\prime}\right)}
$$

if $(\beta)$ and $\left(\beta^{\prime}\right)$ are linked by the following substitutions:

$(\beta) \quad\left(\beta^{\prime}\right)$

(iii) $\left[\begin{array}{ccccc}a+1 & a+1 & c & \\ c & & & b+1 \\ & d & b+1\end{array}\right] \leftrightarrow\left[\begin{array}{ccccc}a & & c+1 & \\ c+1 & & & d+1 \\ & d+1 & b & b\end{array}\right] N \geqq 4$

We have to show that the equalities (87) imply that the coefficients $\varrho_{(\beta)}$ corresponding to all the $(\beta)$ such that the elements of each row sum up to a given integer are equal.

First one observes that all three types of substitutions are such that the sum of elements in any row is not changed. We will show by recurrence that given an arbitrary $(\beta)$ it can be brought with the help of the above substitutions to a canonical form $\left(\beta_{0}\right)$; hence all $\varrho_{(\beta)}$ such that the sum in each row of $(\beta)$ is equal to a given non negative integer are all equal to $\varrho_{\left(\beta_{0}\right)}$ and $\Phi$ is a linear combination of $\Phi_{[\mathrm{m}]}$.

The recurrence starts with $N=2$. We have an arbitrary matrix

$$
(\beta)=\left(\begin{array}{cc}
2 a & c \\
c & 2 b
\end{array}\right) \text { with } 2 a+c=m_{1} \quad 2 b+c=m_{2} .
$$

It is understood that all quantities are non negative integers. Two cases arise $-m_{1}$ and $m_{2}$ both even, then $c$ is necessary even and $(\beta)$ can be brought with the help of (i) to the form

$$
\left(\begin{array}{cc}
m_{1} & 0 \\
0 & m_{2}
\end{array}\right)
$$

$-m_{1}$ and $m_{2}$ both odd then $c$ is also odd and again repeated application of 
(i) leads to

$$
\left(\begin{array}{cc}
m_{1}-1 & 1 \\
1 & m_{2}-1
\end{array}\right) \text {. }
$$

Suppose now $N>2$, with (i) one can reduce the non diagonal terms to be 0 or 1 , then with (ii) one can further reduce the number of off-diagonal terms in each row or column different from zero to be at most one, this exhausts the possible canonical forms if $N$ is equal to 3 ; indeed we have the possibilities:

$$
\begin{aligned}
& m_{1}=2 p_{1} \\
& m_{2}=2 p_{2} \\
& m_{3}=2 p_{3}
\end{aligned} \quad \beta_{0}=\left[\begin{array}{ccc}
2 p_{1} & 0 & 0 \\
0 & 2 p_{2} & 0 \\
0 & 0 & 2 p_{3}
\end{array}\right] ; \begin{aligned}
& m_{1}=2 p_{1}+1 \\
& m_{2}=2 p_{2}+1 \\
& m_{3}=2 p_{3}
\end{aligned} \quad \beta_{0}=\left[\begin{array}{ccc}
2 p_{1} & 1 & 0 \\
1 & 2 p_{2} & 0 \\
0 & 0 & 2 p_{3}
\end{array}\right]
$$

the other two cases with only $m_{2}$ or $m_{1}$ odd are easily deduced from the preceding one.

If $N>3$ we use (iii) to show that the position of the possible 1 in the first row (and the first column) is irrelevant so that according to $m_{1}=2 p_{1}+1$ or $2 p_{1}$, $(\beta)$ can be brought to the form

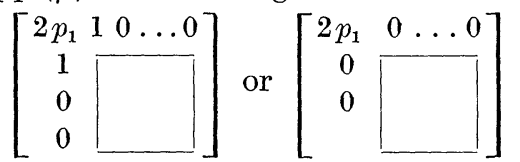

and the box represents an $N-1$ by $N-1$ matrix $\left(\beta^{\prime}\right)$ with $m_{1}^{\prime}=m_{2}-1$ or $m_{2}, m_{3}=m_{3} \ldots, m_{N-1}^{\prime}=m_{N}$ to which the preceding procedure can be applied again. This concludes the proof of the lemma if we remark that the unicity of the decomposition $\Phi=\sum_{[m]} C_{[m]} \Phi_{[m]}$ stems from the linear independance of the functions $\Phi_{[m]}$.

An obvious extension of this result is that we can replace a polynomial $\Phi$ by a function analytic in the neighborhood of $S=0$.

Lemma 7. Let $\Phi(S)$ be analytic in the domain $1-\bar{S} S>0, S^{T}=S$ and $K(i j, l m)$ denote the second order differential operators

$$
K(i j, l m) \equiv K_{i j} K_{l m}-K_{i m} K_{l j} \quad K_{i j}=2^{\delta_{i i}-1} \frac{\partial}{\partial S_{i j}}
$$

if for all values of $i, j, l, m$

$$
K(i j, l m) \Phi(S)=0
$$

then the same holds for $V(k) \Phi(S)$ defined by $(70)$.

Proof. We use the fact that $V^{+}(k) V^{+}\left(k^{\prime}\right)= \pm V^{+}\left(k k^{\prime}\right)$ and the polar decomposition of any element $k \in K_{N}$ as given by lemma 2 to reduce the proof to the case

(i) $k$ is of the form $g=\left(\begin{array}{cc}\bar{U} & 0 \\ 0 & U\end{array}\right), U$ unitary

(ii) $k$ is of the form $d(\theta, 0, \ldots, 0$ ) (see lemma 2 ) since

$$
d\left(\theta_{1}, \theta_{2}, \ldots, \theta_{N}\right)=d\left(\theta_{1}, 0, \ldots, 0\right) d\left(0, \theta_{2}, 0, \ldots, 0\right) d\left(0, \ldots, \theta_{N}\right)
$$

(i) $V^{+}(g) \Phi(S)=(\operatorname{det} U)^{-\frac{1}{2}} \Phi\left(U^{T} S U\right)$. 
Call $S^{\prime}=U^{T} S U, U=\left\{U_{i j}\right\}$, and $K^{\prime}(i j, l m)$ the differential operator corresponding to the variables $\left\{S_{i j}^{\prime}\right\}$ then

$$
K(i j, l m)=\sum_{i^{\prime} j^{\prime} l^{\prime} m^{\prime}} U_{i i^{\prime}} U_{j j^{\prime}} U_{l l^{\prime}} U_{m m^{\prime}} K^{\prime}\left(i^{\prime} j^{\prime}, l^{\prime} m^{\prime}\right)
$$

and the lemma is proved in that case.

(ii) We can write $d(\theta, 0, \ldots, 0), \theta$ real, as

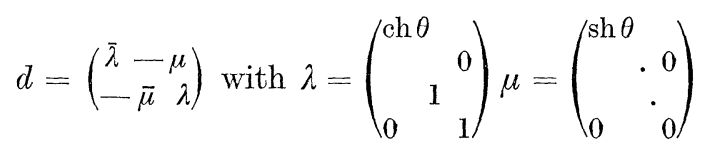

then $S^{\prime}=(\lambda+S \mu)^{-1}(\tilde{\mu}+S \bar{\lambda})$ has the following matrix elements

$$
\begin{gathered}
S_{11}^{\prime}=\frac{\operatorname{ch} \theta S_{11}+\operatorname{sh} \theta}{\operatorname{sh} \theta S_{11}+\operatorname{ch} \theta}, S_{1 \alpha}^{\prime}=\frac{S_{1 \alpha}}{\operatorname{sh} \theta S_{11}+\operatorname{ch} \theta}, \\
S_{\alpha \beta}^{\prime}=\frac{\operatorname{ch} \theta S_{\alpha \beta}+\operatorname{sh} \theta\left(S_{\alpha \beta}-S_{11}-S_{\alpha 1} S_{1 \beta}\right)}{\operatorname{sh} \theta S_{11}+\operatorname{ch} \theta}
\end{gathered}
$$

with $\alpha, \beta$ running from 2 to $N$. The determinant of $\lambda+S \mu$ is equal to $\operatorname{sh} \theta S_{11}+\operatorname{ch} \theta$, we call this quantity $D$. Then the statement of the lemma is deduced from the following equalities, obtained by direct computation where $D$ is considered as the operator $\Phi(S) \rightarrow D \Phi(S)$

$$
\begin{gathered}
D^{\frac{1}{2}} K(11, \alpha \beta) D^{-\frac{1}{2}}=D^{-2}\left[K^{\prime}(11, \alpha \beta)-\operatorname{sh} \theta \sum_{\gamma=2}^{N} S_{1 \gamma} \times\right. \\
\times\left(K^{\prime}(1 \gamma, \alpha \beta)+K^{\prime}\left(\gamma 1, \alpha_{\beta}\right)\right) \\
\left.+\operatorname{sh}^{2} \theta \sum_{\gamma, \delta=2}^{N} S_{1 \gamma} S_{1 \delta} K^{\prime}(\gamma \delta, \alpha \beta)\right] \\
D^{\frac{1}{2}} K(1 \alpha, \beta \gamma) D^{-\frac{1}{2}}=D^{-1}\left[K^{\prime}(1 \alpha, \beta \gamma)-\operatorname{sh} \theta \sum_{\delta=2}^{N} S_{1 \delta} K^{\prime}(\delta \alpha, \beta \gamma)\right] \\
D^{\frac{1}{2}} K(\alpha \beta, \gamma \delta) D^{-\frac{1}{2}}=K^{\prime}(\alpha \beta, \gamma \delta) .
\end{gathered}
$$

All the greek indices run from 2 to $N$ and due to the symmetries of the operators $K(i j, l m)$ we have exhausted all the possibilities. Since

$$
V^{+}(k) \Phi(S)=D^{-\frac{1}{2}} \Phi\left(S^{\prime}\right)
$$

the preceding expansions show that any operator $K(i j, l m)$ applied to $V^{+}(k) \Phi(s)$ is zero if the same is true for $\Phi(S)$.

\section{References}

[1] Bargmann, V.: Commun. Pure Appl. Math. 14, 187 (1961).

[2] Shale: quoted in R. Hermann: Lie groups for physicists. New York: W. A. Benjamin 1965.

[3] Bargmann, V.: Ann. Math. 48, 569 (1947).

[4] Balian, R., C. De Dominicis, and C. Itzykson: Nucl. Phys. 67, 609 (1965). 